\title{
STABILIZATION OF GALERKIN APPROXIMATIONS OF TRANSPORT EQUATIONS BY SUBGRID MODELING
}

\author{
JEAN-LuC GUERMOND ${ }^{1}$
}

\begin{abstract}
This paper presents a stabilization technique for approximating transport equations. The key idea consists in introducing an artificial diffusion based on a two-level decomposition of the approximation space. The technique is proved to have stability and convergence properties that are similar to that of the streamline diffusion method.
\end{abstract}

AMS Subject Classification. 35L50, 65N30.

Received: February 20, 1998. Revised: May 31, 1999.

\section{INTRODUCTION}

The objective of this paper is to present a stabilization technique for solving convection dominated convection/diffusion/reaction equations. The stability on the convection derivative is obtained by introducing an artificial diffusion based on a hierarchical decomposition of the approximation space. The method is proved to have the same stability and convergence properties as those of the streamline diffusion method of BrooksHughes [8]. The reader is referred to Johnson-Nävert-Pitkäranta [20] for a thorough convergence and stability analysis of the streamline diffusion method.

We consider in $\Omega \subset \mathbb{R}^{d}$ the following convection/diffusion/reaction equation

$$
\sigma u+\beta \cdot \nabla u-\epsilon \Delta u=f,
$$

supplemented with reasonable boundary conditions. Coefficient $\epsilon$ is positive, $\sigma$ is a bounded positive function, and $\beta$ is a smooth vector field. It is known that approximating this equation by means of a standard Galerkin technique yields a solution that loses its $H^{1}$ stability when $\epsilon \rightarrow+0$ unless $\|\beta\| h / \epsilon$ is of $\mathcal{O}(1)$. This phenomenon is characterized by severe node to node oscillations of the numerical solution which are generally amplified by nonlinearities.

On possible cure to recover some sort of stability within the framework of the Galerkin technique consists in devising an upwind approximation of the convection derivative by means of the method of characteristics (see [11], [24] or [27]). This technique is quite efficient but we shall not discuss its qualities here.

Another possibility consists in using test functions like $\beta \cdot \nabla \phi_{h}$ to cope with the dominant convection derivative. This idea is at the origin of the Petrov-Galerkin and Least-Square methods; see e.g. [8]. The reader is referred to [25] for an introduction to these techniques and to [10] for a unifying review on this class of methods. Actually, it is shown in [7] that some of these techniques can, at least formally, be recast into the

Keywords and phrases. Linear hyperbolic equations, hierarchical finite elements, stabilization, subgrid modeling, artificial viscosity.

${ }^{1}$ LIMSI, CNRS-UPR 3152, BP 133, 91403 Orsay, France. e-mail: guermond@limsi.fr 
classical Galerkin framework provided some suitably shaped bubble functions are added to the approximation spaces. Furthermore, it is proved in [4] that there always exists a set of internal bubble functions that reproduce the effects of Galerkin Least Square techniques; the exact shape of the bubble functions, though, is in general unknown. This idea, however, makes clear that the stabilization of convection dominated equations is linked to the smallest scales of the approximation space, and that the small scales in question may adequately be modeled by bubble functions. Some authors have considered the practical possibility of stabilizing convection dominated equations by means of bubble functions and subgrid scales; see for instance [19] or [15].

In parallel to the efforts of applied mathematicians to stabilize convection equations, the idea of modeling subgrid scales to avoid unrealistic numerical wiggles has been explored in the Computational Fluid Dynamics community for some times. One tenet of some researchers in this field is that numerical wiggles are due to an "energy accumulation" at the smallest scales of the grid. In other words, since in general the smallest scales of the grid are not fine enough for the viscous dissipation to be effective (i.e. $\|\beta\| \Delta x / \epsilon \gg 1$ ), the flow produces small scales that accumulate at the finest grid scale. This problem is linked by some authors to turbulence and the energy cascade of Kolmogorov. Since, due to limited computer resources, the dissipation scales are generally out of reach, the "energy accumulation" is usually avoided by introducing some artificial dissipation mechanism. These techniques are usually referred to as subgrid eddy viscosity models. Many models of this type have been proposed, and most of them stem from that of Smagorinsky [26], or are closely related to the nonlinear viscosity models of Ladyzhenskaya [21]. The most sophisticated techniques consist in determining dynamically the constants involved in the dissipation models as in [16]. The dynamic control of the constants is generally based on the notion of scale separation; that is, it is assumed that there exists large scales and small scales, the information being transferred from the fine mesh to the coarse one by means of a filter. All these concepts are in general heuristic and, to the authors knowledge, there exists no proof of stability or convergence based on dynamical models.

The idea of scale separation has been rigorously exploited by the Nonlinear Galerkin Method developed by Foias-Manley-Temam [13] and implemented within the finite element framework by Marion-Temam [22]. The reader is also referred to [1] for a two-grid implementation of the NLGM and a novel interpretation of NLGM as a two-level postprocessing of the Galerkin method by García-Archilla-Novo-Titi [28]. The algorithm consists in splitting the unknown into coarse and small scales and in simplifying the dynamics of the small scales. Some of the ideas and notations of the present paper have their roots in the Nonlinear Galerkin method; though, the scope of the present work is less ambitious than NGLM for it is restricted to linear scalar hyperbolic equations.

The objective of this paper is to present a stabilization technique that is based on the principles shared by all the methods referred to above; namely: Galerkin approximation, scale separation, and artificial dissipation of small scales. The content of the paper is organized as follows. In Section 2, we introduce the basic ideas of the method; in particular, we give rationales for introducing scale separation and we propose a dissipation mechanism. In Section 3 we build an abstract theory and we prove quasi-optimal convergence results on a scalar linear hyperbolic equation. We also prove superconvergence results on special meshes. We show in Sections 4 and 5, by giving admissible examples, that the abstract framework developed in Section 3 is not void. The last section in devoted to some concluding remarks.

\section{Position of the PROBlem}

\subsection{Preliminaries}

Let $\Omega$ be an open bounded connected subset of $\mathbb{R}^{d}$. The boundary of $\Omega$ is assumed to be piecewise $C^{1}$; the outward normal on $\Gamma$ is denoted by $n$. Let $\beta$ be a vector field in $\mathcal{C}^{1}(\bar{\Omega})^{d}$. To give a weak sense to derivatives of type $\beta \cdot \nabla u$, we introduce the vector space

$$
H(\beta ; \Omega)=\left\{v \in \mathrm{L}^{2}(\Omega) \mid(\beta \cdot \nabla v) \in \mathrm{L}^{2}(\Omega)\right\}
$$


and we define the semi-norm $|v|_{1, \beta}=\|\beta \cdot \nabla v\|_{0} . H(\beta ; \Omega)$ is a Hilbert space when equipped with the norm $\|v\|_{1, \beta}=\left(\|v\|_{0}^{2}+\|\beta \cdot \nabla v\|_{0}^{2}\right)^{1 / 2}$. Furthermore, let us define

$$
\Gamma^{-}=\{x \in \Gamma \mid \beta(x) \cdot n(x)<0\}, \quad \Gamma^{+}=\{x \in \Gamma \mid \beta(x) \cdot n(x)>0\} \cdot
$$

It is well known that functions of $H(\beta ; \Omega)$ have traces on $\Gamma^{-}$and $\Gamma^{+}$in $L_{l o c}^{2}\left(\Gamma^{-} ;|\beta \cdot n|\right)$ and $L_{l o c}^{2}\left(\Gamma^{+} ; \beta \cdot n\right)$ respectively. Furthermore, it can be shown that

$$
H_{0}(\beta ; \Omega)=\left\{v \in H(\beta ; \Omega) \mid v_{\mid \Gamma^{-}}=0\right\}
$$

is a closed subspace of $H(\beta ; \Omega)$; see e.g. [5] or Chap. XXI in [12] for more details.

\subsection{A model problem}

To avoid unnecessary calculations and to pinpoint the most important arguments, we shall consider hereafter the following problem: for $f \in \mathrm{L}^{2}(\Omega)$, find $u \in H_{0}(\beta ; \Omega)$ so that

$$
\left\{\begin{array}{l}
\sigma u+\beta \cdot \nabla u=f \\
u_{\mid \Gamma^{-}}=0
\end{array}\right.
$$

The function $\sigma(x)$ is in $L^{\infty}(\Omega)$ and we set $\sigma_{1}=\|\sigma\|_{0, \infty}$. The viscous diffusion term is not considered for it would not contribute significantly to the present analysis but would rather blur it. All that is said afterwards can be extended to take into account the viscous diffusion (see [18]).

To simplify the notations, we denote by $A: H_{0}(\beta ; \Omega) \longrightarrow \mathrm{L}^{2}(\Omega)$ the operator so that $A v=\sigma v+\beta \cdot \nabla v$ for all $v$ in $H_{0}(\beta ; \Omega)$, and we introduce the bilinear form $a: H_{0}(\beta ; \Omega) \times \mathrm{L}^{2}(\Omega) \longrightarrow \mathbb{R}$ so that

$$
a(u, v)=\int_{\Omega} A(u) v=\int_{\Omega} \sigma u v+\int_{\Omega}(\beta \cdot \nabla u) v .
$$

The weak formulation of problem (2.4) consists in

$$
\left\{\begin{array}{l}
\text { Find } u \text { in } H_{0}(\beta ; \Omega) \text { so that, } \\
a(u, \phi)=\int_{\Omega} f \phi, \quad \forall \phi \in \mathrm{L}^{2}(\Omega) .
\end{array}\right.
$$

Now, we make the two following assumptions:

(H1) $A: H_{0}(\beta ; \Omega) \longrightarrow \mathrm{L}^{2}(\Omega)$ is an isomorphism.

(H2) $a$ is positive.

Given Banach's closed range theorem, the hypothesis (H1) is equivalent to assuming that there is $\gamma>0$ so that

$$
\left\{\begin{array}{l}
\inf _{v \in H_{0}(\beta ; \Omega)} \sup _{\phi \in \mathrm{L}^{2}(\Omega)} \frac{a(v, \phi)}{\|v\|_{1, \beta}\|\phi\|_{0}} \geq \gamma, \\
\forall \phi \in \mathrm{L}^{2}(\Omega), \quad\left(\forall v \in H_{0}(\beta ; \Omega), a(v, \phi)=0\right) \Rightarrow(\phi=0) .
\end{array}\right.
$$

It is hereafter understood that quantities like $\sup _{x} \phi(x) /\|x\|$ or $\inf x_{x} \phi(x) /\|x\|$, where $x$ spans a normed space, assume $x \neq 0$.

Remark 2.1. The hypothesis (H1) holds if there is $c>0$ so that $2 \sigma(x)-(\nabla \cdot \beta) \geq c$ for a.e. $x$ in $\Omega$. If $\sigma=0$ and $\nabla \cdot \beta=0,(\mathrm{H} 1)$ holds if $\beta$ is a filling field; that is, for a.e. $x$ in $\Omega$ there is a backward characteristics starting from $x$ that reaches $\Gamma^{-}$in finite time. The reader is referred to [3] for other details on this problem. 
As an easy consequence of the hypothesis (H1), we infer:

Proposition 2.1. Problem (2.6) has a unique solution $u$ and

$$
\gamma\|u\|_{1, \beta} \leq\|f\|_{0} .
$$

Remark 2.2. The key idea of the present paper is that a good discrete approximation of problem (2.6) should satisfy the discrete counterpart of (2.7).

\subsection{The scale separation}

The goal of this section is to give heuristic rationales for scale separation.

Note that problem (2.6) is essentially a Petrov-Galerkin problem; that is, the solution space and the test function space are different. Let us denote by $X_{H}$ and $X_{h}$ internal finite dimensional approximation spaces of $H_{0}(\beta ; \Omega)$ and $\mathrm{L}^{2}(\Omega)$ respectively. From the standard approximation theory, it is clear that the couple $\left(X_{H}, X_{h}\right)$ would yield optimal error bounds if $\operatorname{dim}\left(X_{h}\right)=\operatorname{dim}\left(X_{H}\right)$ and if the inf-sup condition (2.7) was uniformly satisfied at the discrete level; that is,

$$
\exists c>0, \forall(H, h), \forall v_{H} \in X_{H}, \quad \sup _{\phi_{h} \in X_{h}} \frac{\int_{\Omega} a\left(v_{H}, \phi_{h}\right)}{\left\|\phi_{h}\right\|_{0}} \geq c\left\|v_{H}\right\|_{1, \beta} .
$$

Unfortunately, for Galerkin approximations $\left(i . e . X_{H}=X_{h}\right.$ ), this condition is in general not satisfied uniformly, since the stability constant $c$ usually depends on the meshsize. For instance, for $\Omega=] 0,1[$ and $\beta \cdot \nabla=\mathrm{d} / \mathrm{d} x$ we have:

Theorem 2.1. Let $\mathcal{T}_{H}$ be a uniform partition of $\Omega$, and let $X_{H} \in H_{0}(\beta ; \Omega)$ be the $\mathbb{P}_{1}$ finite element space based on $\mathcal{T}_{H}$. The are $c_{1}>0$ and $c_{2}>0$ so that

$$
c_{1} H \leq \inf _{v \in X_{H}} \sup _{\phi \in X_{H}} \frac{\int_{0}^{1} v^{\prime}(x) \phi(x) \mathrm{d} x}{\|v\|_{1}\|\phi\|_{0}} \leq c_{2} H
$$

As a result, the Galerkin approximation of the problem $u^{\prime}(x)=f(x)$ cannot be optimal. On the other hand, if one chooses $X_{h}=A\left(X_{H}\right)$, one recovers the Least-Square theory, and the discrete inf-sup condition above is satisfied with $c=\gamma$.

Now let us draw some conclusions. From the weak formulation (2.6), we infer that the discrete problem should involve two approximation spaces $\left(X_{H}, X_{h}\right)$ that should satisfy condition (2.9) to yield stability in the graph norm of $A$. Moreover, the inclusion $H_{0}(\beta ; \Omega) \subset \mathrm{L}^{2}(\Omega)$, implies that $X_{H}$ should be a subspace of $X_{h}$. Furthermore, from the theory developed in $[4,7]$, we deduce that if $X_{H}$ is a standard finite element space, then only bubble-like small scale functions are missing for the Galerkin approximation to work properly. As a result, we are led to introduce an additional discrete space $X_{h}^{H}$ composed of the missing small scales, which have yet to be clearly identified, so that by setting

$$
X_{h}=X_{H} \oplus X_{h}^{H},
$$

the couple $\left(X_{H}, X_{h}\right)$ satisfies the inf-sup condition (2.9) uniformly. Another pragmatic argument for introducing the additional space $X_{h}^{H}$ is that since the inf-sup condition is not satisfied uniformly by using $X_{H}$ as solution and test space, a sure way of satisfying this condition uniformly is to enlarge the test space. We shall hereafter refer to $X_{H}$ as the resolved scale space whereas $X_{h}^{H}$ will be referred to as the subgrid scale space. The reason for these names will be made clear at the end of the paper. 


\subsection{A simple dissipation model}

In general, the Petrov-Galerkin approximation of (2.6) based on the couple $\left(X_{H}, X_{h}\right)$ is not very easy to implement since the two spaces are not of the same dimension (there are more equations than unknowns). The Galerkin approximation of (2.6) based on $X_{h}$ is not realistic too, unless the small scale space $X_{h}^{H}$ is finely tuned according to the Baiocchi et al. theory [4]. The alternative strategy that we propose consists in adopting a Galerkin approximation based on $X_{h}$. Thanks to the inf-sup condition (2.9) we shall be able to control the resolve scales of the solution, but to control its subgrid scales we introduce an artificial diffusion mechanism as follows:

$$
c_{\mathrm{diff}} \int_{\Omega}\left(\beta \cdot \nabla u_{h}^{H}\right)\left(\beta \cdot \nabla v_{h}^{H}\right), \quad \text { or } \quad c_{\mathrm{diff}} \int_{\Omega}\left(\nabla u_{h}^{H}\right) \cdot\left(\nabla v_{h}^{H}\right),
$$

where $u_{h}^{H}$ is the subgrid scale of the approximate solution, $v_{h}^{H}$ is the subgrid scale of the test function, and $c_{\mathrm{diff}}$ is a small coefficient yet to be defined.

Now we have the two ingredients that are necessary for building our Galerkin approximation: a separation of scales and an artificial dissipation mechanism that acts only on the smallest scales of the grid. It is the purpose of the next section to formalize these ideas and to prove that they yield satisfactory stability and convergence properties.

\section{The Discrete SETting}

\subsection{The abstract framework}

To build a discrete approximation of $u$, we introduce $X_{H}$ and $X_{h}$, two finite dimensional subspaces of $H_{0}(\beta ; \Omega)$. The indices $H$ and $h$ denote two positive parameters tending to zero.

We assume also that $X_{H}$ has the following approximation property: there is $k \geq 1$ and $c>0$ so that, for all $v \in H^{l}(\Omega), l \leq k+1$, there is $I_{H} v \in X_{H}$ so that

$$
\left\|v-I_{H} v\right\|_{s} \leq c H^{l-s}\|v\|_{l}, \quad s \in\{0,1\}
$$

From now on, $c$ denotes a generic constant that does not depend on $(H, h)$ and the value of which may change on different occurrences.

The couple $\left(X_{H}, X_{h}\right)$ is assumed to satisfy the following discrete inf-sup condition:

$$
\forall u_{H} \in X_{H} ; \quad \sup _{v_{h} \in X_{h}} \frac{a\left(u_{H}, v_{h}\right)}{\left\|v_{h}\right\|_{0}} \geq c\left\|u_{H}\right\|_{1, \beta} .
$$

Furthermore, we assume that $X_{H} \subset X_{h}$, and there is a linear operator $P_{H}: X_{h} \longrightarrow X_{H}$ that is stable with respect to the $L^{2}$ norm:

$$
\exists c>0, \forall(H, h), \forall v_{h} \in X_{h} \quad\left\|P_{H} v_{h}\right\|_{0} \leq c\left\|v_{h}\right\|_{0}
$$

For further references, we denote $X_{h}^{H}=\left(1-P_{H}\right) X_{h}$. It is clear that $X_{h}=X_{H} \oplus X_{h}^{H}$. For all $v_{h}$ in $X_{h}$ we set

$$
v_{H}=P_{H} v_{h}, \quad \text { and } \quad v_{h}^{H}=v_{h}-v_{H} .
$$

In addition, we assume that $X_{h}$ has the following inverse stability property: there is $c>0$, independent of $(H, h)$, so that

$$
\forall v_{h} \in X_{h}, \quad\left\|\nabla v_{h}\right\|_{0} \leq c H^{-1}\left\|v_{h}\right\|_{0}
$$


Remark 3.1. If $X_{H}$ and $X_{h}$ are finite element spaces for which $H$ and $h$ denote the meshsizes, this property implies that $H$ and $h$ are of the same order; that is, there are $c_{1}>0, c_{2}>0$ so that: $c_{1} H \leq h \leq c_{2} H$. In practice, one usually have $h \approx H / 2$ (see examples of Sect. 5).

The stability hypothesis (3.3) on $P_{H}$ together with the inverse stability property (3.4) yields:

Lemma 3.1. There is $c>0$ so that for all $v_{h} \in X_{h}$,

$$
\left\|\nabla v_{h}^{H}\right\|_{0} \leq c H^{-1}\left\|v_{h}\right\|_{0}
$$

Proof.

$$
\left\|\nabla v_{h}^{H}\right\|_{0} \leq\left\|\nabla v_{h}\right\|_{0}+\left\|\nabla v_{H}\right\|_{0} \leq c H^{-1}\left(\left\|v_{h}\right\|_{0}+\left\|v_{H}\right\|_{0}\right) \leq c H^{-1}\left\|v_{h}\right\|_{0}
$$

\subsection{The discrete problem}

Now we introduce the bilinear form associated with the artificial viscous dissipation. Let $b_{h}: X_{h}^{H} \times X_{h}^{H} \longrightarrow \mathbb{R}$ satisfying the following continuity and coercivity properties: there are $c_{b}>0$ and $c_{B}>0$ so that

$$
\left\{\begin{array}{l}
b_{h}\left(v_{h}^{H}, v_{h}^{H}\right) \geq c_{b} H\left\|v_{h}^{H}\right\|_{b}^{2}, \\
b_{h}\left(v_{h}^{H}, w_{h}^{H}\right) \leq c_{B} H\left\|v_{h}^{H}\right\|_{b}\left\|w_{h}^{H}\right\|_{b},
\end{array}\right.
$$

where the norm $\|\cdot\|_{b}$ is such that there are constants $c_{e 1}>0$ and $c_{e 2}>0$ so that

$$
c_{e 1}\left\|v_{h}^{H}\right\|_{1, \beta} \leq\left\|v_{h}^{H}\right\|_{b} \leq c_{e 2}\left\|v_{h}^{H}\right\|_{1} .
$$

Note that many choices for $b_{h}$ are possible in principle. In particular, the following two choices are admissible

$$
b_{h}\left(v_{h}^{H}, w_{h}^{H}\right)=\left\{\begin{array}{l}
c_{b} H \int_{\Omega}\left(\beta \cdot \nabla v_{h}^{H}\right)\left(\beta \cdot \nabla w_{h}^{H}\right) \\
c_{b} H \int_{\Omega}\left(\nabla v_{h}^{H}\right) \cdot\left(\nabla w_{h}^{H}\right) .
\end{array}\right.
$$

The discrete problem we consider hereafter is

$$
\left\{\begin{array}{l}
\text { Find } u_{h} \text { in } X_{h} \text { so that } \\
a\left(u_{h}, v_{h}\right)+b_{h}\left(u_{h}^{H}, v_{h}^{H}\right)=\int_{\Omega} f v_{h}, \quad \forall v_{h} \in X_{h}
\end{array}\right.
$$

Owing to the coercivity property of $b_{h}$ and condition (3.2), this problem has a unique solution. This formulation can be seen as the mathematical counterpart of the heuristic principle of dynamical subgrid modelling which consists in damping only the small scales. 


\subsection{Error analysis}

The main convergence result of this paper is:

Theorem 3.1. If $u$, the solution to (2.6), is in $H^{k+1}(\Omega)$, then the discrete solution $u_{h}$ to (3.9) satisfies

$$
\left\|u-u_{h}\right\|_{1, \beta}+\left\|u_{h}^{H}\right\|_{b} \leq c H^{k}\|u\|_{k+1} .
$$

And if a is $L^{2}$ coercive,

$$
\left\|u-u_{h}\right\|_{0} \leq c H^{k+1 / 2}\|u\|_{k+1}
$$

Proof. To simplify the presentation of the proof, we introduce some notations. Let $I_{H} u$ be one interpolate of $u$ that satisfies (3.1). We set

$$
\eta_{h}=u-I_{H} u, \quad \text { and } \quad e_{h}=I_{H} u-u_{h} .
$$

The function $\eta_{h}$ is the interpolation error, whereas $e_{h}$ is the approximation error. Note that we have $u-u_{h}=$ $\eta_{h}+e_{h}$.

The equation that controls $e_{h}$ is obtained by subtracting (3.9) to (2.6):

$$
\forall v_{h} \in X_{h}, \quad a\left(e_{h}, v_{h}\right)-b_{h}\left(u_{h}^{H}, v_{h}^{H}\right)=-a\left(\eta_{h}, v_{h}\right) .
$$

Since $X_{H}$ is invariant by the projection $P_{H}$ and $P_{H}$ is linear, we infer

$$
\begin{aligned}
u_{h}^{H} & =u_{h}-P_{H} u_{h}, \\
& =u_{h}-I_{H} u-P_{H}\left(u_{h}-I_{H} u\right), \\
& =-e_{h}+P_{H} e_{h}, \\
& =-e_{h}^{H} .
\end{aligned}
$$

As a result, the equation that controls $e_{h}$ can be recast into the form

$$
\forall v_{h} \in X_{h}, \quad a\left(e_{h}, v_{h}\right)+b_{h}\left(e_{h}^{H}, v_{h}^{H}\right)=-a\left(\eta_{h}, v_{h}\right) .
$$

By taking $e_{h}$ as test function and by using the coercivity property (3.6) we obtain

$$
a_{s}\left(e_{h}, e_{h}\right)+c_{b} H\left|e_{h}^{H}\right|_{b}^{2} \leq-a\left(\eta_{h}, e_{h}\right),
$$

where $a_{s}(v, \phi)=(a(v, \phi)+a(\phi, v)) / 2$ is the symmetric part of $a$. Since $a$ is positive (cf. hypothesis (H2)), $a_{s}$ is a symmetric positive bilinear form. To control the right-hand side of the inequality above, we proceed as follows:

$$
\begin{aligned}
-a\left(\eta_{h}, e_{h}\right) & =a\left(e_{h}, \eta_{h}\right)-2 a_{s}\left(e_{h}, \eta_{h}\right) \\
& \leq\|a\|\left\|e_{h}\right\|_{1, \beta}\left\|\eta_{h}\right\|_{0}+\gamma a_{s}\left(e_{h}, e_{h}\right)+c_{\gamma} a_{s}\left(\eta_{h}, \eta_{h}\right) \\
& \leq\|a\|\left\|e_{h}\right\|_{1, \beta}\left\|\eta_{h}\right\|_{0}+\gamma a_{s}\left(e_{h}, e_{h}\right)+c_{\gamma}\left\|\eta_{h}\right\|_{1, \beta}\left\|\eta_{h}\right\|_{0},
\end{aligned}
$$

where we have used the inequality $a_{s}(v, \phi) \leq \gamma a_{s}(v, v)^{2}+a_{s}(\phi, \phi)^{2} / 4 \gamma$ which is valid for all positive constant $\gamma$, for $a_{s}$ is symmetric positive. Hereafter $\gamma$ denotes a generic positive constant that can be chosen as small as needed and $c_{\gamma}$ is a generic positive constant that depends on $\gamma$. The value of $\gamma$ and $c_{\gamma}$ may change at each occurrence. Note that the term $\left\|e_{h}\right\|_{1, \beta}$ that appears in the right-hand side still needs to be bounded from above. 
It is a this point that the discrete inf-sup condition plays its role. Owing to inequality (3.2) and Lemma 3.1, we have

$$
\begin{aligned}
c\left\|e_{H}\right\|_{1, \beta} & \leq \sup _{v_{h} \in X_{h}} \frac{a\left(e_{H}, v_{h}\right)}{\left\|v_{h}\right\|_{0}} \\
& \leq\|a\|\left(\left\|e_{h}^{H}\right\|_{1, \beta}+\left\|\eta_{h}\right\|_{1, \beta}\right)+c_{B} H\left\|e_{h}^{H}\right\|_{b} \sup _{v_{h} \in X_{h}} \frac{\left\|v_{h}^{H}\right\|_{b}}{\left\|v_{h}\right\|_{0}}, \\
& \leq c\left(\left\|e_{h}^{H}\right\|_{b}+\left\|\eta_{h}\right\|_{1, \beta}\right) .
\end{aligned}
$$

By using the triangular inequality, $\left\|e_{h}\right\|_{1, \beta} \leq\left\|e_{H}\right\|_{1, \beta}+\left\|e_{h}^{H}\right\|_{1, \beta}$, we obtain

$$
\left\|e_{h}\right\|_{1, \beta} \leq c\left(\left\|e_{h}^{H}\right\|_{b}+\left\|\eta_{h}\right\|_{1, \beta}\right)
$$

Finally for $-a\left(\eta_{h}, e_{h}\right)$ we obtain the following bound

$$
\begin{aligned}
-a\left(\eta_{h}, e_{h}\right) & \leq c\left(\left\|e_{h}^{H}\right\|_{b}+\left\|\eta_{h}\right\|_{1, \beta}\right)\left\|\eta_{h}\right\|_{0}+\gamma a_{s}\left(e_{h}, e_{h}\right)+c_{\gamma}\left\|\eta_{h}\right\|_{1}\left\|\eta_{h}\right\|_{0} \\
& \leq \gamma c_{b} H\left\|e_{h}^{H}\right\|_{b}^{2}+\gamma a_{s}\left(e_{h}, e_{h}\right)+c_{\gamma}\left\|\eta_{h}\right\|\left(H^{-1}\left\|\eta_{h}\right\|_{0}+\left\|\eta_{h}\right\|_{1}\right)
\end{aligned}
$$

By substituting this bound into (3.12), we end up with

$$
(\alpha-\gamma) a_{s}\left(e_{h}, e_{h}\right)+(1-\gamma) c_{b} H\left|e_{h}^{H}\right|_{b}^{2} \leq c_{\gamma}\left\|\eta_{h}\right\|_{0}\left(H^{-1}\left\|\eta_{h}\right\|_{0}+\left\|\eta_{h}\right\|_{1}\right)
$$

By choosing $\gamma=1 / 2$ and by using the bound (3.13), we obtain

$$
a_{s}\left(e_{h}, e_{h}\right)+c_{b} H\left(\left\|e_{h}\right\|_{1, \beta}^{2}+\left\|e_{h}^{H}\right\|_{b}^{2}\right) \leq c\left\|\eta_{h}\right\|_{0}\left(H^{-1}\left\|\eta_{h}\right\|_{0}+\left\|\eta_{h}\right\|_{1}\right) .
$$

Moreover, if $a$ is $L^{2}$ coercive, we have $a_{s}\left(e_{h}, e_{h}\right) \geq \alpha\left\|e_{h}\right\|_{0}^{2}$; that is,

$$
\left\|e_{h}\right\|^{2} \leq c\left\|\eta_{h}\right\|_{0}\left(H^{-1}\left\|\eta_{h}\right\|_{0}+\left\|\eta_{h}\right\|_{1}\right)
$$

The desired bounds follow readily.

Remark 3.2. The above convergence result is identical to the one that can be obtained when approximating (2.6) by means of the Streamline Diffusion technique (see [20]).

Remark 3.3. Note that if the bilinear form $b_{h}$ is built on the streamline diffusion model, then the present stabilization technique does not introduce artificial cross-wind diffusion. However, the above convergence result also implies that the isotropic diffusion model can be used without loss of convergence. This model may be helpful to avoid cross-wind oscillations that can appear when approximating very stiff problems.

\subsection{Superconvergence results}

As noted in [29], superconvergence is often observed when using the Streamline Diffusion technique to solve (2.6). It is shown in [29] that this surprising effect is the consequence of using special structured meshes. It is the purpose of this section to show that the analysis of [29] can be extended to the present sub-grid modeling technique.

Let us introduce a new definition: For all $v$ in $H(\beta ; \Omega)$ we define the following mesh-dependent semi-norm.

$$
|v|_{\beta, 0, h}=\sup _{w_{h} \in X_{h}} \frac{\left(\beta \cdot \nabla v, w_{h}\right)}{\left\|w_{h}\right\|_{0}} .
$$


Now, we assume that there are $c$ and $s$ independent of $(H, h), 0 \leq s \leq 1$, so that $u$ is in $H^{k+s}(\Omega)$ and satisfies the estimate:

$$
\left|u-I_{H} u\right|_{\beta, 0, h} \leq c H^{k+s}\|u\|_{k+s} .
$$

Owing to the approximation property (3.1) of $I_{H}$, this inequality holds obviously true for $s=0$. If $X_{H}$ is a finite element based approximation space and provided the finite element grid satisfies some restrictive conditions, (3.15) can be proved to hold with $s>0$ by using techniques similar to those of Zhou [29]. The reader is referred to $[23,29]$ for more details on the conditions that the grid must satisfy.

Theorem 3.2. Assume $a$ is $\mathrm{L}^{2}(\Omega)$ coercive. If $u$, the solution of (2.6), is in $H^{k+s}(\Omega)$ and if the superinterpolation result (3.15) holds with $s \geq 1 / 2$, then the discrete solution $u_{h}$ of (3.9) satisfies

$$
\left\|u-u_{h}\right\|_{0}+c_{b} H^{1 / 2}\left|u_{h}^{H}\right|_{b} \leq c H^{k+s}\|u\|_{k+s} .
$$

Proof. We proceed as in the proof of theorem 3.1, but to bound from above $\left|a\left(\eta_{h}, e_{h}\right)\right|$, we use the superinterpolation property as follows

$$
\begin{aligned}
-a\left(\eta_{h}, e_{h}\right) & =-\left(\sigma \eta_{h}, e_{h}\right)-\left(\beta \cdot \nabla \eta_{h}, e_{h}\right) \\
& \leq \gamma\left\|e_{h}\right\|_{0}^{2}+c_{\gamma}\left\|\eta_{h}\right\|_{0}^{2}+\left|\eta_{h}\right|_{\beta, 0, h}\left\|e_{h}\right\|_{0} \\
& \leq \gamma\left\|e_{h}\right\|_{0}^{2}+c_{\gamma}\left(\left\|\eta_{h}\right\|_{0}^{2}+\left|\eta_{h}\right|_{\beta, 0, h}^{2}\right) .
\end{aligned}
$$

By using $a\left(e_{h}, e_{h}\right) \geq \alpha\left\|e_{h}\right\|_{0}^{2}$, by substituting the bound above into (3.12), and by choosing $\gamma=\alpha / 2$, we obtain

$$
\alpha\left\|e_{h}\right\|_{0}^{2}+c_{b} H\left|e_{h}^{H}\right|_{b}^{2} \leq c\left(\left|\eta_{h}\right|_{\beta, 0, h}^{2}+\left\|\eta_{h}\right\|_{0}^{2}\right) .
$$

The final estimate follows readily from this bound and the super-interpolation property (3.15).

\section{Checking the $L^{2}$ StABility And the WeAK INF-SuP CONDition}

By explicitly building admissible couples $\left(X_{H}, X_{h}\right)$, we show in this section that the abstract framework developed above is not void.

\subsection{Criteria for checking the weak inf-sup condition}

A classical criterion for verifying that an inf-sup-type condition is satisfied is as follows (cf. e.g. [14] or [17]):

Lemma 4.1. Assume that there is a projection $\Pi_{h}: \mathrm{L}^{2}(\Omega) \longrightarrow X_{h}$ and there is $c_{\Pi_{h}}>0$ so that for all $u_{H} \in X_{H}$ and $v \in \mathrm{L}^{2}(\Omega),\left\|\Pi_{h} v\right\|_{0} \leq c_{\Pi_{h}}\|v\|_{0}$ and $\int_{\Omega}\left(\beta \cdot \nabla u_{H}\right) v=\int_{\Omega}\left(\beta \cdot \nabla u_{H}\right) \Pi_{h} v$, then

$$
\inf _{u_{H} \in X_{H}} \sup _{v_{h} \in X_{h}} \frac{\int_{\Omega}\left(\beta \cdot \nabla u_{H}\right) v_{h}}{\left|u_{H}\right|_{1, \beta}\left\|v_{h}\right\|_{0}} \geq c_{\Pi_{h}}^{-1} .
$$

Proof. Let $u_{H}$ be a function in $X_{H}$.

$$
\begin{aligned}
\sup _{v_{h} \in X_{h}} \frac{\int_{\Omega}\left(\beta \cdot \nabla u_{H}\right) v_{h}}{\left\|v_{h}\right\|_{0}} & \geq \sup _{v \in \mathrm{L}^{2}(\Omega)} \frac{\int_{\Omega}\left(\beta \cdot \nabla u_{H}\right) \Pi_{h} v}{\left\|\Pi_{h} v\right\|_{0}}, \\
& \geq c_{\Pi_{h}}^{-1} \sup _{v \in \mathrm{L}^{2}(\Omega)} \frac{\int_{\Omega}\left(\beta \cdot \nabla u_{H}\right) v}{\|v\|_{0}}, \\
& \geq c_{\Pi_{h}}^{-1}\left\|\beta \cdot \nabla u_{H}\right\|_{0} .
\end{aligned}
$$


The result follows easily.

The following lemma gives a sufficient condition for (3.2) to hold if $\sigma \neq 0$ and if $a$ is $L^{2}$ coercive; i.e. $a(v, v) \geq \alpha\|v\|_{0}^{2}$ :

Lemma 4.2. Assume $X_{H} \subset X_{h}$ and there are $c_{\beta}>0$ and $c_{\delta} \geq 0$, both independent of $(H, h)$, so that

$$
\forall v_{H} \in X_{H}, \quad \sup _{\phi_{h} \in X_{h}} \frac{\int_{\Omega}\left(\beta \cdot \nabla v_{H}\right) \phi_{h}}{\left\|\phi_{h}\right\|_{0}} \geq c_{\beta}\left|v_{H}\right|_{1, \beta}-c_{\delta}\left\|v_{H}\right\|_{0},
$$

then, there is $c=\left(c_{\beta} \alpha\right) /\left(c_{\beta}^{2}+\left(\alpha+\sigma_{1}+c_{\delta}\right)^{2}\right)^{1 / 2}$ so that(3.2) holds.

Proof. It is clear that owing to the $L^{2}$ coercivity and the hypothesis $X_{H} \subset X_{h}$ we have

$$
\sup _{\phi \in X_{h}} \frac{a\left(v_{H}, \phi_{h}\right)}{\left\|\phi_{h}\right\|_{0}} \geq \alpha\left\|v_{H}\right\|_{0}
$$

Furthermore, we have

$$
\begin{aligned}
\sup _{\phi_{h} \in X_{h}} \frac{a\left(v_{H}, \phi_{h}\right)}{\left\|\phi_{h}\right\|_{0}} & \geq \sup _{\phi_{h} \in X_{h}} \frac{\int_{\Omega}\left(\beta \cdot \nabla v_{H}\right) \phi}{\left\|\phi_{h}\right\|_{0}}-\sup _{\phi_{h} \in X_{h}} \frac{\int_{\Omega} \sigma v_{H} \phi_{h}}{\left\|\phi_{h}\right\|_{0}} \\
& \geq\left|v_{H}\right|_{1, \beta}-\sigma_{1}\left\|v_{H}\right\|_{0}, \\
& \geq\left|v_{H}\right|_{1, \beta}-\frac{\sigma_{1}}{\alpha} \sup _{\phi_{h} \in X_{h}} \frac{a\left(v_{H}, \phi_{h}\right)}{\left\|\phi_{h}\right\|_{0}}
\end{aligned}
$$

The desired inequality follows easily.

We finish this section by giving a perturbation result that will prove useful in the following:

Lemma 4.3. Let $\beta_{1}$ and $\beta_{2}$ be two vector fields so that $\left\|\beta_{1}-\beta_{2}\right\|_{0, \infty} \leq c H$, where $c \geq 0$ is independent of $(H, h)$. Assume that there are $c_{\beta_{1}}>0$ and $c_{\delta_{1}} \geq 0$ so that

$$
\forall u_{H} \in X_{H}, \quad \sup _{v_{h} \in X_{h}} \frac{\int_{\Omega}\left(\beta_{1} \cdot \nabla u_{H}\right) v_{h}}{\left\|v_{h}\right\|_{0}} \geq c_{\beta_{1}}\left|u_{H}\right|_{1, \beta_{1}}-c_{\delta_{1}}\left\|u_{H}\right\|_{0},
$$

then, there are $c_{\beta_{2}}>0$ and $c_{\delta_{2}} \geq 0$ so that

$$
\forall u_{H} \in X_{H}, \quad \sup _{v_{h} \in X_{h}} \frac{\int_{\Omega}\left(\beta_{2} \cdot \nabla u_{H}\right) v_{h}}{\left\|v_{h}\right\|_{0}} \geq c_{\beta_{2}}\left|u_{H}\right|_{1, \beta_{2}}-c_{\delta_{2}}\left\|u_{H}\right\|_{0} .
$$

Proof. Let $u_{H}$ be a function in $X_{H}$.

$$
\begin{aligned}
\sup _{v_{h} \in X_{h}} \frac{\int_{\Omega}\left(\beta_{2} \cdot \nabla u_{H}\right) v_{h}}{\left\|v_{h}\right\|_{0}} & \geq-\sup _{v_{h} \in X_{h}} \frac{\int_{\Omega}\left(\left(\beta_{2}-\beta_{1}\right) \cdot \nabla u_{H}\right) v_{h}}{\left\|v_{h}\right\|_{0}}+\sup _{v \in X_{h}} \frac{\int_{\Omega}\left(\beta_{1} \cdot \nabla u_{H}\right) v_{h}}{\left\|v_{h}\right\|_{0}}, \\
& \geq-\left\|\beta_{2}-\beta_{1}\right\|_{0, \infty}\left|u_{H}\right|_{1}+c_{\beta_{1}}\left\|\beta_{1} \cdot \nabla u_{H}\right\|_{0}-c_{\delta_{1}}\left\|u_{H}\right\|_{0}, \\
& \geq-\left(c+c_{\delta_{1}}\right)\left\|u_{H}\right\|_{0}-c_{\beta_{1}}\left\|\left(\beta_{1}-\beta_{2}\right) \cdot \nabla u_{H}\right\|_{0}+c_{\beta_{1}}\left\|\beta_{2} \cdot \nabla u_{H}\right\|_{0}, \\
& \geq-\left(c+c_{\delta_{1}}\right)\left\|u_{H}\right\|_{0}-c_{\beta_{1}}\left\|\beta_{1}-\beta_{2}\right\|_{0, \infty}\left|u_{H}\right|_{1}+c_{\beta_{1}}\left\|\beta_{2} \cdot \nabla u_{H}\right\|_{0}, \\
& \geq c_{\beta_{2}}\left|u_{H}\right|_{1, \beta_{2}}-c_{\delta_{2}}\left\|u_{H}\right\|_{0} .
\end{aligned}
$$

The proof is complete. 


\section{2. $\boldsymbol{L}^{\mathbf{2}}$ stability of the scale decomposition}

In this section, we propose, for quite general finite element spaces, a scale decomposition technique that is $L^{2}$ stable. For the sake of simplicity, we assume throughout this section that $\Omega$ is a $\mathbb{R}^{d}$-polyhedron and $\mathcal{T}_{H}$ is a regular, quasi-uniform mesh of $\Omega$ composed of affine simplexes, $\left(T_{H}\right)$. The reference simplex is denoted by $\hat{T}$ and $F_{H}: T_{H} \longrightarrow \hat{T}$ is the one to one affine mapping that maps $T_{H}$ onto $\hat{T}$. For any function defined on $T_{H}$, we denote $\hat{v}=v\left(F_{H}^{-1}\right)$.

Let $\hat{\phi}_{1}, \ldots, \hat{\phi}_{k_{H}}$ be a family of $k_{H}$ linearly independent functions in $H^{1}(\hat{T}) \cap C^{0}(\overline{\hat{T}})$. We define $X_{H}$ as follows:

$$
X_{H}=\left\{v_{H} \in H^{1}(\Omega) \cap H_{0}(\beta ; \Omega) \mid v_{H}\left(F_{H}^{-1}\right)_{\mid T_{H}} \in \operatorname{span}\left(\hat{\phi}_{1}, \ldots, \hat{\phi}_{k_{H}}\right), \forall T_{H} \in \mathcal{T}_{H}\right\} .
$$

Let $\hat{\psi}_{1}, \ldots, \hat{\psi}_{k_{h}}$ be a family of $k_{h}$ linearly independent functions in $H^{1}(\hat{T}) \cap C^{0}(\overline{\hat{T}})$ so that the family $\left(\hat{\phi}_{1}, \ldots, \hat{\phi}_{k_{H}}\right.$, $\hat{\psi}_{1}, \ldots, \hat{\psi}_{k_{h}}$ ) is linearly independent. We define $X_{h}^{H}$ as follows:

$$
X_{h}^{H}=\left\{v_{h}^{H} \in H^{1}(\Omega) \cap H_{0}(\beta ; \Omega) \mid v_{h}^{H}\left(F_{H}^{-1}\right)_{\mid T_{H}} \in \operatorname{span}\left(\hat{\psi}_{1}, \ldots, \hat{\psi}_{k_{h}}\right), \forall T_{H} \in \mathcal{T}_{H}\right\} .
$$

Owing to the linear independence of the basis functions, it is clear that $X_{H} \cap X_{h}^{H}=\emptyset$. As a result, we can define $X_{h}=X_{H} \oplus X_{h}^{H}$. Furthermore, we have

Proposition 4.1. Provided the basis functions $\left(\hat{\phi}_{1}, \ldots, \hat{\phi}_{k_{H}}, \hat{\psi}_{1}, \ldots, \hat{\psi}_{k_{h}}\right)$ are linearly independent, the decomposition $X_{h}=X_{H} \oplus X_{h}^{H}$ is $L^{2}$ stable.

Proof. Let $v_{h}$ be a function in $X_{h}$ and $v_{h}=v_{H}+v_{h}^{H}$ be its decomposition. We have

$$
\left\|v_{h}\right\|_{0}^{2}=\left\|v_{H}+v_{h}^{H}\right\|_{0}^{2}=\left\|v_{H}\right\|_{0}^{2}+\left\|v_{h}^{H}\right\|_{0}^{2}+2 \sum_{T_{H}} \int_{T_{H}} v_{H} v_{h}^{H}
$$

Let us define

$$
C(\hat{T})=\sup _{\hat{v}_{H}, \hat{v}_{h}^{H}} \frac{\int_{\hat{T}} \hat{v}_{H} \hat{v}_{h}^{H}}{\left\|\hat{v}_{H}\right\|_{0, \hat{T}}\left\|\hat{v}_{h}^{H}\right\|_{0, \hat{T}}}
$$

Note that $C(\hat{T})$ does not depend on $(H, h)$. Since the couple $\left(\hat{v}_{H}, \hat{v}_{h}^{H}\right)$ spans a finite dimensional vector space, a compacity argument implies that the supremum is reached for some $\left(\hat{w}_{H}, \hat{w}_{h}^{H}\right)$. Since $\hat{w}_{H}$ and $\hat{w}_{h}^{H}$ are linearly independent, we infer that $\int_{\hat{T}} \hat{w}_{H} \hat{w}_{h}^{H}<\left\|\hat{w}_{H}\right\|_{0, \hat{T}}\left\|\hat{w}_{h}^{H}\right\|_{0, \hat{T}}$; as a consequence, we have

$$
C(\hat{T})<1 .
$$

Furthermore, by using the relation $\int_{T_{H}} v_{H} v_{h}^{H}=\frac{\operatorname{meas}\left(T_{H}\right)}{\operatorname{meas}(\hat{T})} \int_{\hat{T}} \hat{v}_{H} \hat{v}_{h}^{H}$, we infer

$$
\begin{aligned}
\int_{T_{H}} v_{H} v_{h}^{H} & \leq C(\hat{T}) \frac{\operatorname{meas}\left(T_{H}\right)}{\operatorname{meas}(\hat{T})}\left\|\hat{v}_{H}\right\|_{0, \hat{T}}\left\|\hat{v}_{h}^{H}\right\|_{0, \hat{T}} \\
& \leq C(\hat{T})\left\|v_{H}\right\|_{0, T_{H}}\left\|v_{h}^{H}\right\|_{0, T_{H}} .
\end{aligned}
$$

This inequality yields

$$
\begin{aligned}
\left\|v_{h}\right\|_{0}^{2} & \geq\left\|v_{H}\right\|_{0}^{2}+\left\|v_{h}^{H}\right\|_{0}^{2}-2 C(\hat{T})\left(\sum_{T_{H}} \int_{T_{H}} v_{H}^{2}\right)^{1 / 2}\left(\sum_{T_{H}} \int_{T_{H}}\left(v_{h}^{H}\right)^{2}\right)^{1 / 2} \\
& \geq(1-C(\hat{T}))\left\|v_{H}\right\|_{0}^{2}+(1-C(\hat{T}))\left\|v_{h}^{H}\right\|_{0}^{2} .
\end{aligned}
$$


Since $C(\hat{T})<1$, the stability of the operator $P_{H}: v_{h} \longmapsto v_{H}$ follows readily.

Remark 4.1. The arguments developed above can be extended to nonaffine mappings and more general finite elements by using more sophisticated compacity arguments.

\section{3. $\mathbb{P}_{\mathbf{1}}$ interpolation + Bubble}

It is shown in Brezzi et al. [7] that the $\mathbb{P}_{1}$ Galerkin technique is equivalent to a Galerkin Least Square approximation provided bubble functions are added to the $\mathbb{P}_{1}$ finite element space and $\beta$ is piecewise constant. The purpose of this section is to shed new light on this result and to prove that, for quite general vector fields $\beta$, it is possible to build a $\mathbb{P}_{1}$-based couple $\left(X_{H}, X_{h}\right)$ that satisfies all the conditions introduced in Section 3.

We define $X_{H}$ as follows

$$
X_{H}=\left\{v_{H} \in H^{1}(\Omega) \cap H_{0}(\beta ; \Omega) \mid v_{H \mid T_{H}} \in \mathbb{P}_{1}\left(T_{H}\right), \forall T_{H} \in \mathcal{T}_{H}\right\} .
$$

Let $\hat{\psi}$ be a bubble function defined on the reference simplex $\hat{T}$; more precisely, we introduce $\hat{\psi} \in H_{0}^{1}(\hat{T})$ with $0 \leq \hat{\psi} \leq 1$. Bubble functions have been introduced initially to build stable approximations of the Stokes equations (cf. e.g. $[2,9])$. We denote $\psi_{h}=\hat{\psi}\left(F_{H}\right)$, where $F_{H}: T_{H} \longrightarrow \hat{T}$ is the one to one affine mapping that maps $T_{H}$ onto $\hat{T}$. We define $c_{\psi}=\int_{\hat{T}} \hat{\psi}^{2} /$ meas $(\hat{T})$. One easily verifies that

$$
\left\{\begin{array}{l}
\int_{T_{H}} \psi_{h}^{2}=c_{\psi} \operatorname{meas}\left(T_{H}\right), \\
c_{\psi} \operatorname{meas}\left(T_{H}\right) \leq \int_{T_{H}} \psi_{h} \leq \operatorname{meas}\left(T_{H}\right) .
\end{array}\right.
$$

We set

$$
X_{h}^{H}=\left\{v_{h}^{H} \in H^{1}(\Omega) \cap H_{0}(\beta ; \Omega) \mid v_{H \mid T_{H}}^{h} \in \operatorname{span}\left(\psi_{h}\right), \forall T_{H} \in \mathcal{T}_{H}\right\} .
$$

Owing to Proposition 4.1, the decomposition $X_{h}=X_{H} \oplus X_{h}^{H}$ is $L^{2}$ stable. Furthermore, if $\beta$ is piecewise constant, we have the following result.

Theorem 4.1. If $\beta$ is piecewise constant on each simplex $T_{H}$ of $\mathcal{T}_{H}$, there is $c_{\beta}>0$ independent of $(H, h)$, $c_{\beta}=c_{\psi}^{1 / 2}$, so that

$$
\inf _{u_{H} \in X_{H}} \sup _{v_{h} \in X_{h}} \frac{\int_{\Omega}\left(\beta \cdot \nabla u_{H}\right) v_{h}}{\left|u_{H}\right|{ }_{1, \beta}\left\|v_{h}\right\|_{0}} \geq c_{\beta} .
$$

Proof. First, we define a linear operator $\Pi_{h}: \mathrm{L}^{2}(\Omega) \longrightarrow X_{h}$. Let $v$ be in $\mathrm{L}^{2}(\Omega)$ and define $\Pi_{h} v \in X_{h}^{H}$ so that

$$
\Pi_{h} v_{\mid T_{H}}=\frac{\int_{T_{H}} v}{\int_{T_{H}} \psi_{h}} \psi_{h}
$$

Note that $\Pi_{h} v$ is such that $\int_{T_{H}} v=\int_{T_{H}} \Pi_{h} v$. Furthermore, we have

$$
\begin{aligned}
\left\|\Pi_{h} v\right\|_{0}^{2} & =\sum_{T_{H}}\left(\int_{T_{H}} v\right)^{2}\left(\int_{T_{H}} \psi_{h}\right)^{-2} \int_{T_{H}} \psi_{h}^{2} \\
& \leq \sum_{T_{H}} \operatorname{meas}\left(T_{H}\right)\|v\|_{0, T_{H}}^{2}\left(c_{\psi} \operatorname{meas}\left(T_{H}\right)\right)^{-2} c_{\psi} \operatorname{meas}\left(T_{H}\right) \\
& \leq c_{\psi}^{-1}\|v\|_{0}^{2} .
\end{aligned}
$$



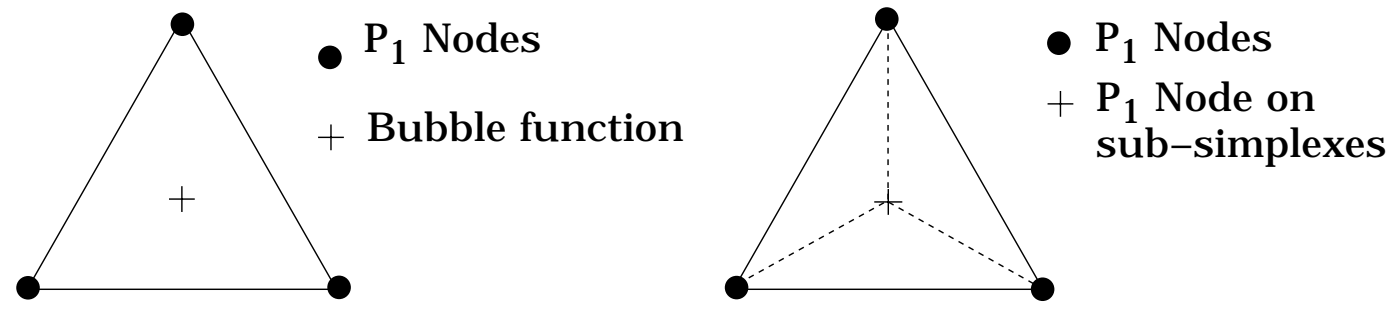

Figure 1. Two representations of the small-scale space $X_{h}^{H}$ for $\mathbb{P}_{1}$ interpolation. Left: the bubble function is $3^{d+1} \lambda_{1} \ldots \lambda_{d+1}$, where $\lambda_{i}$ is the barycentric coordinate associated with node $i$; Right: a node is inserted at the barycenter of $\hat{T}$ and $\hat{T}$ is divided into $d+1 \mathbb{P}_{1}$ subsimplexes.

As a result $\Pi_{h}$ is $L^{2}$ stable, and the stability constant does not depend on $(H, h)$.

Let $u_{H}$ be in $X_{H}$. Since $X_{H}$ is composed of $\mathbb{P}_{1}$ finite elements and $\beta$ is piecewise constant, we infer that $\left(\beta \cdot \nabla u_{H}\right)$ is piecewise $\mathbb{P}_{0}$. As a consequence, one easily verifies that

$$
\int_{\Omega}\left(\beta \cdot \nabla u_{H}\right)\left(v-\Pi_{h} v\right)=0
$$

The conclusion is a consequence of Lemma 4.1.

For a general vector field $\beta$ we deduce:

Theorem 4.2. If $\beta$ is in $C^{1}(\bar{\Omega})$, there are $c_{\beta}>0$ and $c_{\delta} \geq 0$, both independent of $(H, h)$, so that

$$
\forall u_{H} \in X_{H}, \quad \sup _{v_{h} \in X_{h}} \frac{\int_{\Omega}\left(\beta \cdot \nabla u_{H}\right) v_{h}}{\left\|v_{h}\right\|_{0}} \geq c_{\beta}\left|u_{H}\right|_{1, \beta}-c_{\delta}\left\|u_{H}\right\|_{0}
$$

Proof. For each simplex $T_{H}$ we denote by $b_{T_{H}}$ the barycenter of $T_{H}$. Now we define $\beta_{H}$ a piecewise constant function so that $\beta_{H \mid T_{H}}=\beta\left(b_{T_{H}}\right)$. Since $\Omega$ is bounded, we have

$$
\left\|\beta_{H}-\beta\right\|_{0, \infty} \leq c H\|\beta\|_{C^{1}(\bar{\Omega})} .
$$

The desired result is a consequence of Lemma 4.3 and Theorem 4.1.

Remark 4.2. Does (4.8) hold with $c_{\delta}=0$ ?

We finish this section by showing in Figure 1 two possible representations of the small-scale space $X_{h}^{H}$.

\subsection{Two-level $\mathbb{P}_{\mathbf{1}}$ interpolation in $\mathbf{2 D}$}

For the sake of simplicity, we assume in this section that the space is two-dimensional. The purpose of this section is to build a two-level $\mathbb{P}_{1}$ setting that satisfies the hypotheses of Section 3 .

As in the previous section, we define the resolved scale space $X_{H}$ as follows

$$
X_{H}=\left\{v_{H} \in H^{1}(\Omega) \cap H_{0}(\beta ; \Omega) \mid v_{H \mid T_{H}} \in \mathbb{P}_{1}\left(T_{H}\right), \forall T_{H} \in \mathcal{T}_{H}\right\} .
$$

Now let us build the subgrid scale space $X_{h}^{H}$. From each triangle $T_{H} \in \mathcal{T}_{H}$, we create 4 new triangles by connecting the middle of the three edges of $T_{H}$. Let us denote $h=H / 2$, and define $\mathcal{T}_{h}$ the resulting new triangulation. For each macro-triangle $T_{H}$ we denote by $\mathbb{P}$ the set of continuous functions on $T_{H}$ that are piecewise $\mathbb{P}_{1}$ on each subtriangle of $T_{H}$ and vanish at the three vertices of $T_{H}$. Now we set

$$
X_{h}^{H}=\left\{v_{h}^{H} \in H^{1}(\Omega) \cap H_{0}(\beta ; \Omega) \mid v_{h \mid T_{H}}^{H} \in \mathbb{P}, \forall T_{H} \in \mathcal{T}_{H}\right\} .
$$



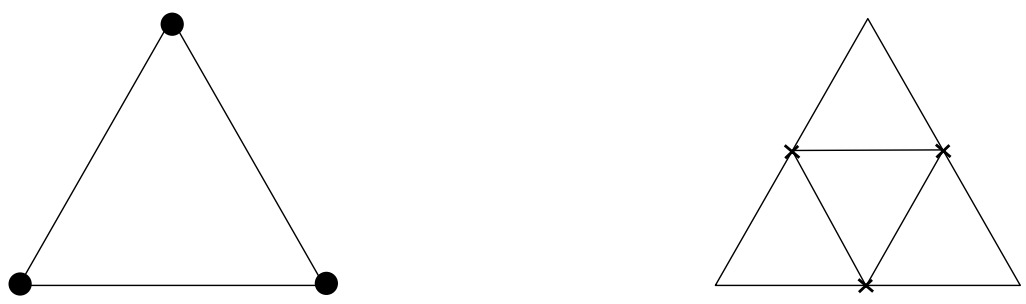

Figure 2. Representations of the finite elements on which the two-level $\mathbb{P}_{1}$ space is built. Left: the resolved scale finite element; Right: the subgrid scale finite element.

It is clear that owing to Proposition 4.1, the decomposition $X_{h}=X_{H} \oplus X_{h}^{H}$ is $L^{2}$ stable. Moreover, $X_{h}$ has the following simple characterization:

$$
X_{h}=\left\{v_{h} \in H^{1}(\Omega) \cap H_{0}(\beta ; \Omega) \mid v_{h \mid T_{h}} \in \mathbb{P}_{1}\left(T_{h}\right), \forall T_{h} \in \mathcal{T}_{h}\right\}
$$

Furthermore, we have:

Theorem 4.3. If $\beta$ is piecewise constant on each simplex $T_{H}$ of $\mathcal{T}_{H}$, there is $c_{\beta}>0$ independent of $(H, h)$, so that

$$
\inf _{u_{H} \in X_{H}} \sup _{v_{h} \in X_{h}} \frac{\int_{\Omega}\left(\beta \cdot \nabla u_{H}\right) v_{h}}{\left|u_{H}\right|_{1, \beta}\left\|v_{h}\right\|_{0}} \geq c_{\beta} .
$$

Furthermore, if $\beta$ is in $C^{1}(\bar{\Omega})$, there are $c_{\beta}>0$ and $c_{\delta} \geq 0$, both independent of $(H, h)$, so that

$$
\forall u_{H} \in X_{H}, \quad \sup _{v_{h} \in X_{h}} \frac{\int_{\Omega}\left(\beta \cdot \nabla u_{H}\right) v_{h}}{\left\|v_{h}\right\|_{0}} \geq c_{\beta}\left|u_{H}\right|_{1, \beta}-c_{\delta}\left\|u_{H}\right\|_{0} .
$$

Proof. The proof is somewhat technical and is reported in [6].

A representation of the two finite elements on which the resolved scale space $X_{H}$ and the subgrid scale space $X_{h}^{H}$ are built is given in Figure 2.

\section{5. $\mathbb{P}_{2}$ interpolation + Bubbles}

Let $X_{H}$ be the $\mathbb{P}_{2}$ finite element space (conformal in $H^{1}(\Omega) \cap H_{0}(\beta ; \Omega)$ ) associated with the triangulation $\mathcal{T}_{H}$ :

$$
X_{H}=\left\{v_{H} \in H^{1}(\Omega) \cap H_{0}(\beta ; \Omega) \mid v_{H \mid T_{H}} \in \mathbb{P}_{2}\left(T_{H}\right), \forall T_{H} \in \mathcal{T}_{H}\right\} .
$$

Let $\hat{\phi}_{1}, \ldots, \hat{\phi}_{d+1}$ be the nodal basis of the $\mathbb{P}_{1}$ Lagrange polynomials associated with $\hat{a}_{1}, \ldots, \hat{a}_{d+1}$ respectively. We denote $\phi_{i, H}=\hat{\phi}_{i}\left(F_{H}\right)$ for $1 \leq i \leq d+1$. Let $\hat{\psi}_{1}, \ldots, \hat{\psi}_{d+1}$ be a family a $d+1$ linearly independent, real-valued functions in $H_{0}^{1}(\hat{T})$. We denote $\psi_{i, h}=\hat{\psi}_{i}\left(F_{H}\right)$ for $1 \leq i \leq d+1$. Let $\hat{\theta}_{1}, \ldots, \hat{\theta}_{k_{H}}$ a basis of $\mathbb{P}_{2}(\hat{T})$. Since $\hat{\psi}_{1}, \ldots, \hat{\psi}_{d+1}$ are in $H_{0}^{1}(\hat{T})$, the family $\hat{\theta}_{1}, \ldots, \hat{\theta}_{k_{H}}, \hat{\psi}_{1}, \ldots, \hat{\psi}_{d+1}$ is necessarily linearly independent. We set

$$
X_{h}^{H}=\left\{v_{h}^{H} \in H^{1}(\Omega) \cap H_{0}(\beta ; \Omega) \mid v_{h \mid T_{H}}^{H} \in \operatorname{span}\left(\psi_{1, h}, \ldots, \psi_{d+1, h}\right), \forall T_{H} \in \mathcal{T}_{H}\right\} .
$$

Now, by setting $X_{h}=X_{H} \oplus X_{h}^{H}$, we obtain a scale decomposition that is $L^{2}$ stable owing to Proposition 4.1.

To guarantee that a discrete inf-sup condition holds, we need to enforce additional hypotheses on the small scale functions. Let $\hat{a}_{1}, \ldots, \hat{a}_{d+1}$ be the nodes of the reference simplex $\hat{T}$. We denote by $R_{i j}$ the symmetry of $\hat{T}$ 
such that $R_{i j}\left(\hat{a}_{i}\right)=\hat{a}_{j}$ and $R_{i j}\left(\hat{a}_{l}\right)=\hat{a}_{l}$ if $l \notin\{i, j\}$. Now, we assume that the functions $\left(\hat{\psi}_{i}\right)_{i=1, \ldots, d+1}$ satisfy the following symmetry properties

$$
\left\{\begin{array}{l}
\hat{\psi}_{i}\left(R_{i j}\right)=\hat{\psi}_{j}, \\
\hat{\psi}_{i}\left(R_{j l}\right)=\hat{\psi}_{i}, \text { if } i \notin\{j, l\}
\end{array}\right.
$$

Note that the $\mathbb{P}_{1}$ basis functions $\left(\hat{\phi}_{i}\right)_{i=1, \ldots, d+1}$ satisfy these relations. As a consequence, we have:

Lemma 4.4. The basis functions $\hat{\phi}_{1}, \ldots, \hat{\phi}_{d+1}, \hat{\psi}_{1}, \ldots, \hat{\psi}_{d+1}$ satisfy the following symmetry relations

$$
\left\{\begin{array}{l}
\int_{\hat{T}} \hat{\phi}_{i} \hat{\psi}_{j}=\int_{\hat{T}} \hat{\phi}_{j} \hat{\psi}_{i}, \\
\int_{\hat{T}} \hat{\phi}_{i} \hat{\psi}_{j}=\int_{\hat{T}} \hat{\phi}_{i} \hat{\psi}_{l}, \text { if } i \notin\{j, l\} \\
\int_{\hat{T}} \hat{\phi}_{i} \hat{\psi}_{i}=\int_{\hat{T}} \hat{\phi}_{j} \hat{\psi}_{j}, \\
\int_{\hat{T}} \hat{\phi}_{i} \hat{\phi}_{j}=\int_{\hat{T}} \hat{\phi}_{l} \hat{\psi}_{m}, \text { if } i \neq j \text { and } l \neq m
\end{array}\right.
$$

Proof. For the first equality we proceed as follows:

$$
\begin{aligned}
\int_{\hat{T}} \hat{\phi}_{i} \hat{\psi}_{j} & =\int_{\hat{T}} \hat{\phi}_{j}\left(R_{i j}\right) \hat{\psi}_{i}\left(R_{i j}\right), \text { since } R_{i j}=R_{j i}, \\
& =\int_{R_{j i}(\hat{T})} \hat{\phi}_{j}\left(R_{i j}\right) \hat{\psi}_{i}\left(R_{i j}\right)\left|\operatorname{det}\left(R_{i j}\right)\right| \text {, since }\left|\operatorname{det}\left(R_{i j}\right)\right|=1 \text {, and } R_{j i}(\hat{T})=\hat{T} \\
& =\int_{\hat{T}} \hat{\phi}_{j} \hat{\psi}_{i} .
\end{aligned}
$$

For the second equality we proceed similarly

$$
\begin{aligned}
\int_{\hat{T}} \hat{\phi}_{i} \hat{\psi}_{j} & =\int_{\hat{T}} \hat{\phi}_{j} \hat{\psi}_{i}, \\
& =\int_{R_{j l}(\hat{T})} \hat{\phi}_{j}\left(R_{l j}\right) \hat{\psi}_{i}\left(R_{l j}\right)\left|\operatorname{det}\left(R_{l j}\right)\right| \\
& =\int_{\hat{T}} \hat{\phi}_{l} \hat{\psi}_{i}, \text { if } i \notin\{j, l\} \\
& =\int_{\hat{T}} \hat{\phi}_{i} \hat{\psi}_{l} .
\end{aligned}
$$

For the third equality we have

$$
\int_{\hat{T}} \hat{\phi}_{i} \hat{\psi}_{i}=\int_{\hat{T}} \hat{\phi}_{j}\left(R_{j i}\right) \hat{\psi}_{j}\left(R_{j i}\right)=\int_{\hat{T}} \hat{\phi}_{j} \hat{\psi}_{j}
$$


For the last equality we have

$$
\begin{aligned}
\int_{\hat{T}} \hat{\phi}_{i} \hat{\psi}_{j} & =\int_{\hat{T}} \hat{\phi}_{i} \hat{\psi}_{m}, \text { if } i \neq j, i \neq m, \\
& =\int_{\hat{T}} \hat{\phi}_{m} \hat{\psi}_{i}, \\
& =\int_{\hat{T}} \hat{\phi}_{m} \hat{\psi}_{l}, \text { if } m \neq i, m \neq l, \\
& =\int_{\hat{T}} \hat{\phi}_{l} \hat{\psi}_{m} .
\end{aligned}
$$

Furthermore, one verifies easily that the equality still holds if $m=i$.

Furthermore, we make the following unrestrictive assumption

$$
\int_{\hat{T}} \hat{\psi}_{i}\left(\hat{\phi}_{i}-\hat{\phi}_{j}\right) \neq 0, \text { if } i \neq j
$$

Lemma 4.5. If $\beta$ is piecewise constant on each simplex of $\mathcal{T}_{h}$, there exists a linear projection operator $\Pi_{h}$ : $\mathrm{L}^{2}(\Omega) \longrightarrow X_{h}$ so that

$$
\forall v \in \mathrm{L}^{2}(\Omega), \forall u_{H} \in X_{H}, \quad \int_{\Omega}\left(\beta \cdot \nabla u_{H}\right)\left(v-\Pi_{H} v\right)=0
$$

and there is $c_{\Pi_{h}}>0$ so that for all $v$ in $\mathrm{L}^{2}(\Omega),\left\|\Pi_{H} v\right\|_{0} \leq c_{\Pi_{h}}\|v\|_{0}$.

Proof. Let us define the matrix $A_{i j}=\int_{\Omega} \hat{\phi}_{i} \hat{\psi}_{j}$, and let us show that $A$ is invertible. $A$ is a $(d+1) \times(d+1)$ real matrix. Owing to lemma 4.4, we infer that $A_{i i}=A_{j j}$ and $A_{i j}=A_{l m}$ if $i \neq j$ and $l \neq m$. Let us denote $p=A_{i i}$ and $q=A_{i j}$ for $i \neq j$; we have

$$
A=\left(\begin{array}{cccccccc}
p & q & \cdot & \cdot & \cdot & \cdot & \cdot & q \\
q & p & \cdot & & & & & \cdot \\
\cdot & \cdot & \cdot & \cdot & & & & \cdot \\
\cdot & & \cdot & \cdot & \cdot & & & \cdot \\
\cdot & & & \cdot & \cdot & \cdot & & \cdot \\
\cdot & & & & \cdot & \cdot & \cdot & \cdot \\
\cdot & & & & \cdot & \cdot & \cdot & q \\
q & \cdot & \cdot & \cdot & \cdot & \cdot & q & p
\end{array}\right) .
$$

Let $D_{d}$ be the determinant of $A$; by developing the determinant with respect to one row, one obtains the following recurrence relation $D_{d}=p D_{d-1}-d q^{2}(p-q)^{d-1}$, from which we deduce by induction $D_{d}=(p-q)^{d}(p-d q)$. Owing to the hypothesis (4.18), $p \neq q$; hence, $D_{d}$ is not zero. As a result, the matrix $A$ is invertible.

Let $v$ be in $\mathrm{L}^{2}(\Omega), T_{H}$ be a simplex of $\mathcal{T}_{H}$, and $\left(c_{1}\left(T_{H}, v\right), \ldots, c_{d+1}\left(T_{H}, v\right)\right)$ be the solution to the following linear system

$$
\sum_{j=1}^{d+1} c_{j}\left(T_{H}, v\right) \int_{T_{H}} \phi_{i, H} \psi_{j, h}=\int_{T_{H}} v \phi_{i, H} .
$$


Since $\int_{T_{H}} \phi_{i, H} \psi_{j, h}$ is equal to $\left(\operatorname{meas}\left(T_{H}\right) / \operatorname{meas}(\hat{T})\right) A_{i j}$, and $A$ is invertible, the system above is invertible. Let us define the following linear operator $\Pi_{h}: \mathrm{L}^{2}(\Omega) \longrightarrow X_{h}$ :

$$
\Pi_{h} v=\sum_{T_{H}} \sum_{j=1}^{d+1} c_{j}\left(T_{H}, v\right) \psi_{j, h} .
$$

Let us show that $\Pi_{h}$ is $\mathrm{L}^{2}(\Omega)$ stable. Since the mapping $F_{H}$ is affine, it is clear that $\left(c_{1}\left(T_{H}, v\right), \ldots, c_{d+1}\left(T_{H}, v\right)\right)$ is also solution to

$$
\sum_{j=1}^{d+1} c_{j}\left(T_{H}, v\right) \int_{\hat{T}} \hat{\phi}_{i} \hat{\psi}_{j, h}=\int_{\hat{T}} \hat{v} \hat{\phi}_{i} .
$$

As a result, there is $\hat{c}$, depending only on $\hat{T}$ and the reference basis, so that

$$
\max _{1 \leq j \leq d+1}\left|c_{j}\left(T_{H}, v\right)\right| \leq \hat{c} \max _{1 \leq i \leq d+1}\left|\int_{\hat{T}} \hat{v} \hat{\phi}_{i}\right| \leq \hat{c} \max _{1 \leq i \leq d+1}\|\hat{v}\|_{0, \hat{T}}\left\|\hat{\phi}_{i}\right\|_{0, \hat{T}} \leq \hat{c}^{\prime}\|\hat{v}\|_{0, \hat{T}} .
$$

Hence, by introducing the notation $c_{\psi}=\int_{\hat{T}} \hat{\psi}_{j}^{2} /$ meas $(\hat{T})$, we have

$$
\begin{aligned}
\left\|\Pi_{h} v\right\|_{0}^{2} & =\sum_{T_{H}}\left\|\Pi_{h} v\right\|_{0, T_{H}}^{2}, \\
& \leq(d+1) \sum_{T_{H}} \sum_{j=1}^{d+1}\left|c_{j}\left(T_{H}, v\right)\right|^{2}\left\|\psi_{j, h}\right\|_{0, T_{H}}^{2}, \\
& \leq(d+1) c_{\psi} \sum_{T_{H}} \max _{1 \leq j \leq d+1}\left|c_{j}\left(T_{H}, v\right)\right|^{2} \operatorname{meas}\left(T_{H}\right), \\
& \leq(d+1) c_{\psi}\left(\hat{c}^{\prime}\right)^{2} \sum_{T_{H}}\|\hat{v}\|_{0, \hat{T}}^{2} \operatorname{meas}\left(T_{H}\right), \\
& \leq(d+1) c_{\psi}\left(\hat{c}^{\prime}\right)^{2} \operatorname{meas}(\hat{T}) \sum_{T_{H}}\|v\|_{0, T_{H}}^{2}, \\
& \leq(d+1) c_{\psi}\left(\hat{c}^{\prime}\right)^{2} \operatorname{meas}(\hat{T})\|v\|_{0}^{2} .
\end{aligned}
$$

Let $u_{H}$ be a function in $X_{H}$. Since $\beta$ is piecewise $\mathbb{P}_{0}$ and and $u_{H}$ is piecewise $\mathbb{P}_{2},\left(\beta \cdot \nabla u_{H}\right)$ is piecewise $\mathbb{P}_{1}$. For a given $T_{H}$ we denote by $\left(\beta \cdot \nabla u_{H}\right)_{\mid T_{H}}=\sum_{i=1}^{d+1} \alpha_{i}\left(T_{H}\right) \phi_{i, H}$ the local $\mathbb{P}_{1}$ decomposition of $\left(\beta \cdot \nabla u_{H}\right)$. Now let us 

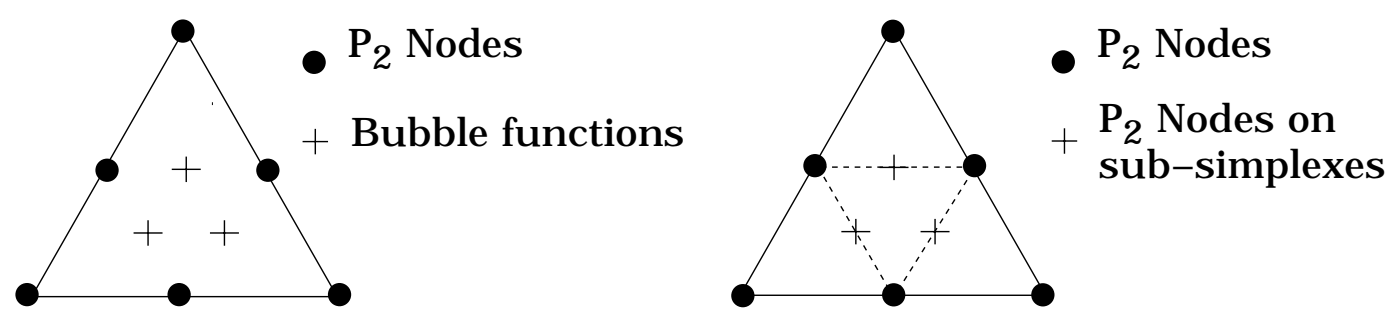

Figure 3. Two representations of the small-scale space $X_{h}^{H}$ for $\mathbb{P}_{2}$ interpolation. Left: $d+1$ bubble functions globally defined on $\hat{T}$; Right: in $2 \mathrm{D}, \hat{T}$ is divided into 4 subtriangles and $3 \mathbb{P}_{2}$ functions are located at $\lambda_{i}=0.5, \lambda_{j}=0.25, j \neq i$.

verify that $\int_{\Omega}\left(\beta \cdot \nabla u_{H}\right)\left(v-\Pi_{h} v\right)=0$ for all $u_{H}$ in $X_{H}$. Owing to the definition of $\Pi_{h} v$ we have

$$
\begin{aligned}
\int_{\Omega}(\beta \cdot \nabla u) \Pi_{h} v & =\sum_{T_{H}} \int_{T_{H}}\left(\beta \cdot \nabla u_{H}\right) \sum_{j=1}^{d+1} c_{j}\left(T_{H}, v\right) \psi_{j, h} \\
& =\sum_{T_{H}} \int_{T_{H}} \sum_{i=1}^{d+1} \alpha_{i}\left(T_{H}\right) \phi_{i, H} \sum_{j=1}^{d+1} c_{j}\left(T_{H}, v\right) \psi_{j, h} \\
& =\sum_{T_{H}} \sum_{i=1}^{d+1} \alpha_{i}\left(T_{H}\right) \sum_{j=1}^{d+1} c_{j}\left(T_{H}, v\right) \int_{T_{H}} \phi_{i, H} \psi_{j, h}, \\
& =\sum_{T_{H}} \sum_{i=1}^{d+1} \alpha_{i}\left(T_{H}\right) \int_{T_{H}} v \phi_{i, H}, \\
& =\sum_{T_{H}} \int_{T_{H}}\left(\sum_{i=1}^{d+1} \alpha_{i}\left(T_{H}\right) \phi_{i, H}\right) v \\
& =\int_{\Omega}\left(\beta \cdot \nabla u_{H}\right) v .
\end{aligned}
$$

The proof is complete.

Now we are in position to prove:

Theorem 4.4. If $\beta$ is in $C^{1}(\bar{\Omega})$, there are $c_{\beta}>0$ and $c_{\delta} \geq 0$ independent of $(H, h)$ so that

$$
\forall u_{H} \in X_{H}, \quad \sup _{v_{h} \in X_{h}} \frac{\int_{\Omega}\left(\beta \cdot \nabla u_{H}\right) v_{h}}{\left\|v_{h}\right\|_{0}} \geq c_{\beta}\left|u_{H}\right|_{1, \beta}-c_{\delta}\left\|u_{H}\right\|_{0} .
$$

Proof. The proof is identical to that of Theorem 4.2.

We finish this section by showing in Figure 3 two possible representations of the subgrid scale space $X_{h}^{H}$.

\subsection{Two-level $\mathbb{P}_{\mathbf{2}}$ interpolation in $2 \mathrm{D}$}

As in Section 4.4, we assume that the space is two-dimensional. We build in this section a two-level $\mathbb{P}_{2}$ setting that satisfies the hypotheses of Section 3.

The resolved scale space $X_{H}$ is defined as in the previous section as follows

$$
X_{H}=\left\{v_{H} \in H^{1}(\Omega) \cap H_{0}(\beta ; \Omega) \mid v_{H \mid T_{H}} \in \mathbb{P}_{2}\left(T_{H}\right), \forall T_{H} \in \mathcal{T}_{H}\right\} .
$$



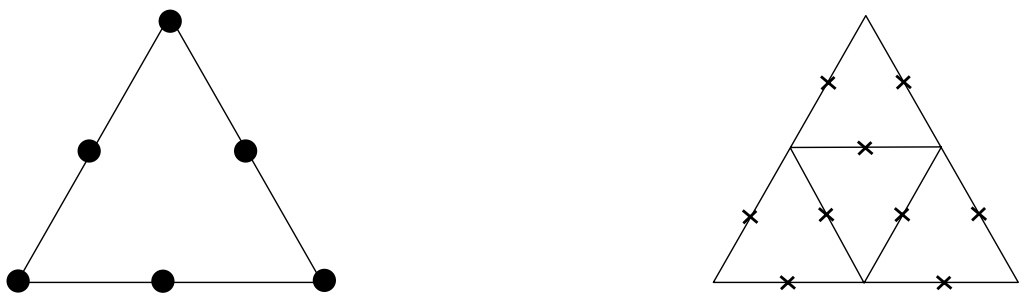

Figure 4. Representations of the finite elements on which $X_{H}$ and $X_{h}^{H}$ are built. Left: the resolved scale finite element; Right: the subgrid scale finite element.

To build the subgrid scale space $X_{h}^{H}$, we proceed as in Section 4.4. We set $h=H / 2$, and we denote by $\mathcal{T}_{h}$ the triangulation that is obtained by dividing each triangle of $\mathcal{T}_{H}$ into four subtriangles. For each triangle $T_{h}$ we denote by $\psi_{1}, \psi_{2}, \psi_{3}$ the three $\mathbb{P}_{2}$ nodal functions associated with the middle of each edges of $T_{h}$. We define the subgrid scale space by

$$
X_{h}^{H}=\left\{v_{h}^{H} \in H^{1}(\Omega) \cap H_{0}(\beta ; \Omega) \mid v_{h \mid T_{h}}^{H} \in \operatorname{span}\left(\psi_{1}, \psi_{2}, \psi_{3}\right), \forall T_{h} \in \mathcal{T}_{h}\right\} .
$$

Owing to Proposition 4.1, the decomposition $X_{h}=X_{H} \oplus X_{h}^{H}$ is $L^{2}$ stable. Moreover, $X_{h}$ has the following simple characterization:

$$
X_{h}=\left\{v_{h} \in H^{1}(\Omega) \cap H_{0}(\beta ; \Omega) \mid v_{h \mid T_{h}} \in \mathbb{P}_{2}\left(T_{h}\right), \forall T_{h} \in \mathcal{T}_{h}\right\}
$$

Furthermore, we have:

Theorem 4.5. If $\beta$ is piecewise constant on each simplex $T_{H}$ of $\mathcal{T}_{H}$, there is $c_{\beta}>0$ independent of $(H, h)$, so that

$$
\inf _{u_{H} \in X_{H}} \sup _{v_{h} \in X_{h}} \frac{\int_{\Omega}\left(\beta \cdot \nabla u_{H}\right) v_{h}}{\left|u_{H}\right|_{1, \beta}\left\|v_{h}\right\|_{0}} \geq c_{\beta}
$$

Furthermore, if $\beta$ is in $C^{1}(\bar{\Omega})$, there are $c_{\beta}>0$ and $c_{\delta} \geq 0$, both independent of $(H, h)$, so that

$$
\forall u_{H} \in X_{H}, \quad \sup _{v_{h} \in X_{h}} \frac{\int_{\Omega}\left(\beta \cdot \nabla u_{H}\right) v_{h}}{\left\|v_{h}\right\|_{0}} \geq c_{\beta}\left|u_{H}\right|_{1, \beta}-c_{\delta}\left\|u_{H}\right\|_{0}
$$

Proof. The proof is simple consequence of Theorem 4.4.

We show in Figure 4 a representation of the two finite elements on which $X_{H}$ and $X_{h}^{H}$ are built.

\section{NumERICAL EXAMPLES}

\subsection{Example 1}

To illustrate the method, we apply it to the following 2D problem:

$$
\left\{\begin{array}{l}
\left.\partial_{y} u=-8 \pi \cos (2 \pi x) \sin (8 \pi y), \quad \text { in } \Omega=\right] 0,1\left[^{2}\right. \\
u_{\mid y=0}=\cos (2 \pi x),
\end{array}\right.
$$



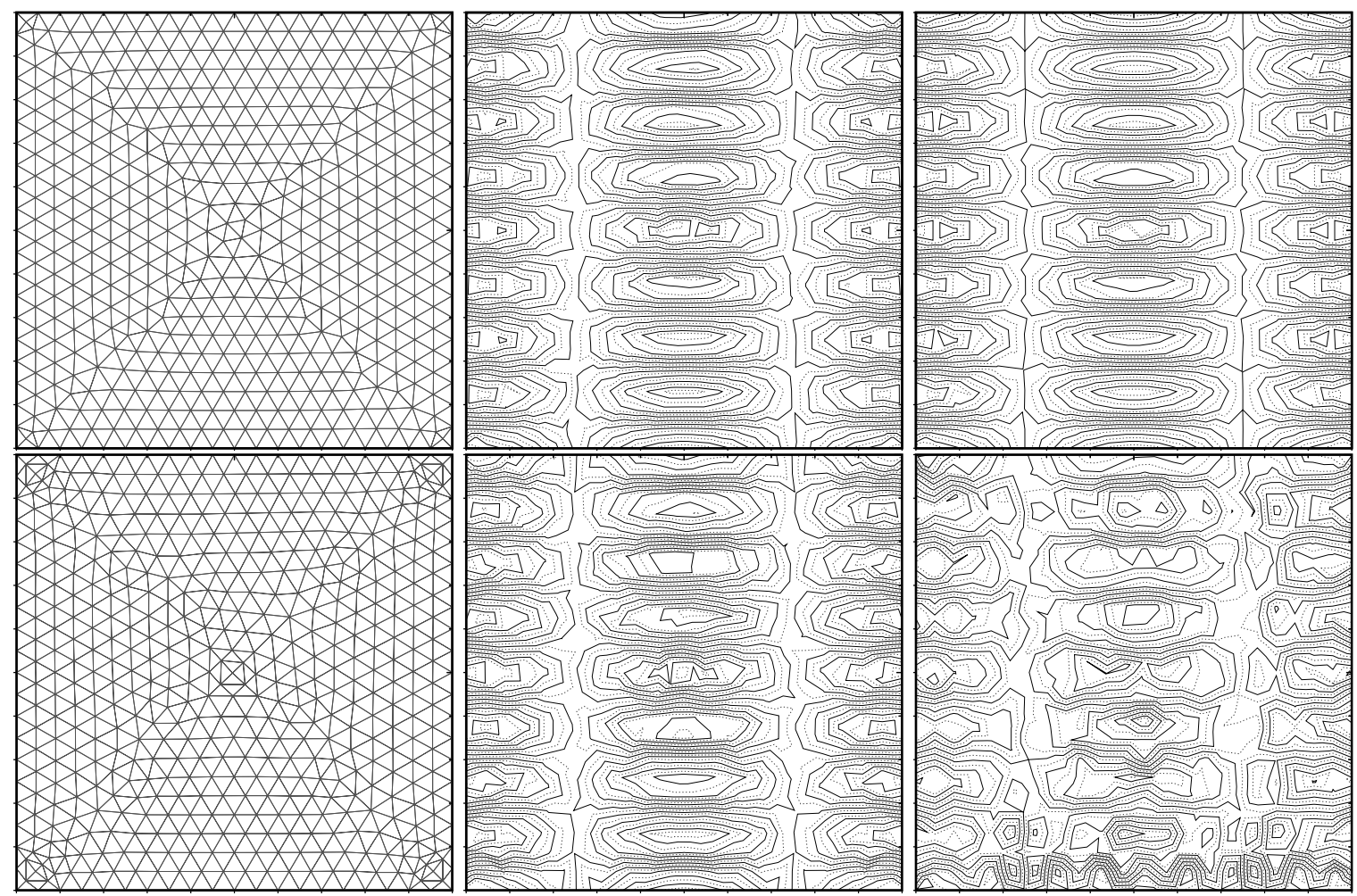

Figure 5. Top left: First mesh for the $\mathbb{P}_{1} /$ bubble solution; top center: stabilized $\mathbb{P}_{1} /$ bubble solution; top right: $\mathbb{P}_{1}$ interpolate of exact solution; bottom left: second mesh for the two-level $\mathbb{P}_{1}$ solution; bottom center: stabilized two-level $\mathbb{P}_{1}$ solution; bottom right: Galerkin solution (equally bad on both meshes).

where $u=\cos (2 \pi x) \cos (8 \pi y)$ is the exact solution. We use $\mathbb{P}_{1}$ interpolation for the resolved scale space $X_{H}$. We make tests with the $\mathbb{P}_{1}$ /bubble framework described in Section 4.3 and with the two-level $\mathbb{P}_{1}$ framework described in Section 4.4. The artificial viscosity is introduced by means of the bilinear form

$$
b_{h}\left(v_{h}^{H}, w_{h}^{H}\right)=\sum_{T_{h} \in \mathcal{T}_{h}} \operatorname{mes}\left(T_{h}\right)^{1 / 2} \int_{T_{h}} \nabla v_{h}^{H} \cdot \nabla w_{h}^{H}
$$

In Figure 5, we have plotted the mesh used for the $\mathbb{P}_{1} /$ bubble stabilization (top left) and the mesh used for the two-level $\mathbb{P}_{1}$ stabilization (bottom left). In the first case there are 932 elements and $507 \mathbb{P}_{1}$ nodes; in the second case there are 952 elements and 517 nodes. In both cases the mesh size $h$ is of order $1 / 20$. In Figure 5 , we have also reported the isovalues of the stabilized $\mathbb{P}_{1} /$ bubble solution (top center); the isovalues of the $\mathbb{P}_{1}$ interpolate of the solution (top right); the isovalues of the stabilized two-level $\mathbb{P}_{1}$ solution (bottom center); the isovalues of the Galerkin solution on the first mesh (the result is equally bad on both meshes) (bottom right). The superiority of the proposed method on the pure Galerkin technique is clear. Note also that the method is very simple to implement since it only consists in adding the perturbation term $b_{h}\left(u_{h}^{H}, v_{h}^{H}\right)$ to the standard Galerkin approximation.

Note also that the linear system associated with the Galerkin solution is so badly conditioned that it is almost impossible to invert it with a preconditioned GMRES algorithm. The only way we found to invert this system 

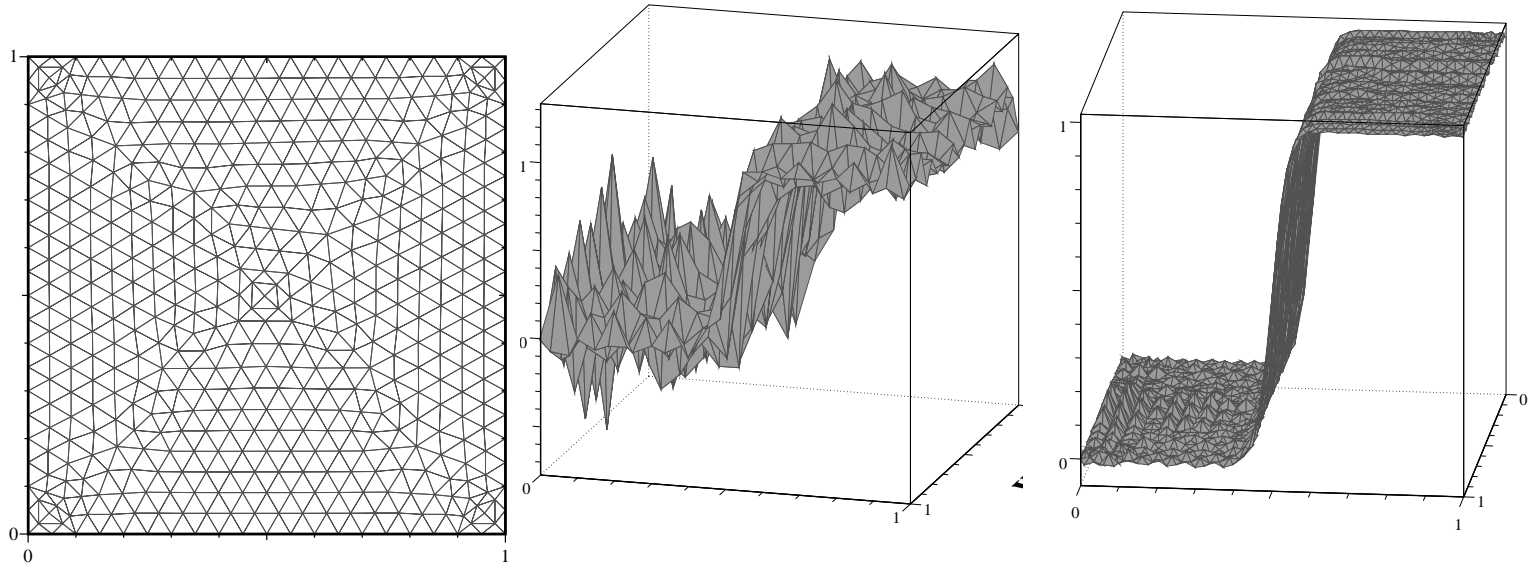

Figure 6. Problem: $\partial_{y} u=\frac{1}{2 \epsilon}\left(1-\left(\tanh \left(\frac{y-0.5)}{\epsilon}\right)^{2}\right)\right.$. Left: two-level finite element mesh; center: $3 \mathrm{D}$ rendering of Galerkin $\mathbb{P}_{1}$ solution; right: $3 \mathrm{D}$ rendering of Galerkin $\mathbb{P}_{2}$ solution.

is to add a small regularizing term so that the equation we actually solve is $0.01 u+\partial_{y} u=f$. The stabilized solutions do not need this regularizing term, for the associated linear systems behave very well.

\subsection{Example 2}

To further illustrate the method, we apply it to the following 2D problem:

$$
\left\{\begin{array}{l}
\left.\partial_{y} u=\frac{1}{2 \epsilon}\left(1-\left(\tanh \left(\frac{y-0.5}{\epsilon}\right)\right)^{2}\right), \quad \text { in } \Omega=\right] 0,1\left[^{2}\right. \\
u_{\mid y=0}=0,
\end{array}\right.
$$

where $u=\frac{1}{2}\left(\tanh \left(\frac{y-0.5}{\epsilon}\right)+1\right)$ is the exact solution with $\epsilon=0.04$. The two-level mesh that we use is composed of 952 elements and 517 nodes and the mesh size $h$ is of order 1/20. This mesh is depicted in Figure 6 (left). A $3 \mathrm{D}$ rendering of the Galerkin solution for $\mathbb{P}_{1}$ interpolation and $\mathbb{P}_{2}$ interpolation is plotted in Figure 6 (center and right). Spurious numerical wiggles are clearly apparent on both solutions, the worst case being the $\mathbb{P}_{1}$ solution.

The stabilized solution is calculated by means of the two-level $\mathbb{P}_{1}$ and two-level $\mathbb{P}_{2}$ interpolation frameworks described in Sections 4.4 and 4.6. The artificial viscosity is the same as in the previous section. The results are reported in Figure 7 . The projection on plane $y=0$ of the $\mathbb{P}_{1}$ and $\mathbb{P}_{2}$ interpolates of the exact solution is plotted on the left of the figure; the projection on plane $y=0$ of the two-level $\mathbb{P}_{1}$ and $\mathbb{P}_{2}$ subgrid viscosity solution is plotted in the center; the projection on plane $y=0$ of the Galerkin $\mathbb{P}_{1}$ and $\mathbb{P}_{2}$ solution is plotted on the right. The capabilities of the subgrid viscosity method described in this paper are clearly demonstrated on this example.

\section{Concluding REMARKS}

A subgrid modeling method for stabilizing Galerkin approximations of transport equations have been presented. The technique is based on a two-level decomposition of the approximation space. The stability on the convection derivative is obtained by introducing an artificial diffusion of the small scales. Stability and convergence properties similar to that of the streamline diffusion method have been proved. In some sense, the present work is a (very) small step towards the theoretical justification of some dynamical subgrid models that are popular in CFD. Of course the present method can be put within the general framework of residual-based 

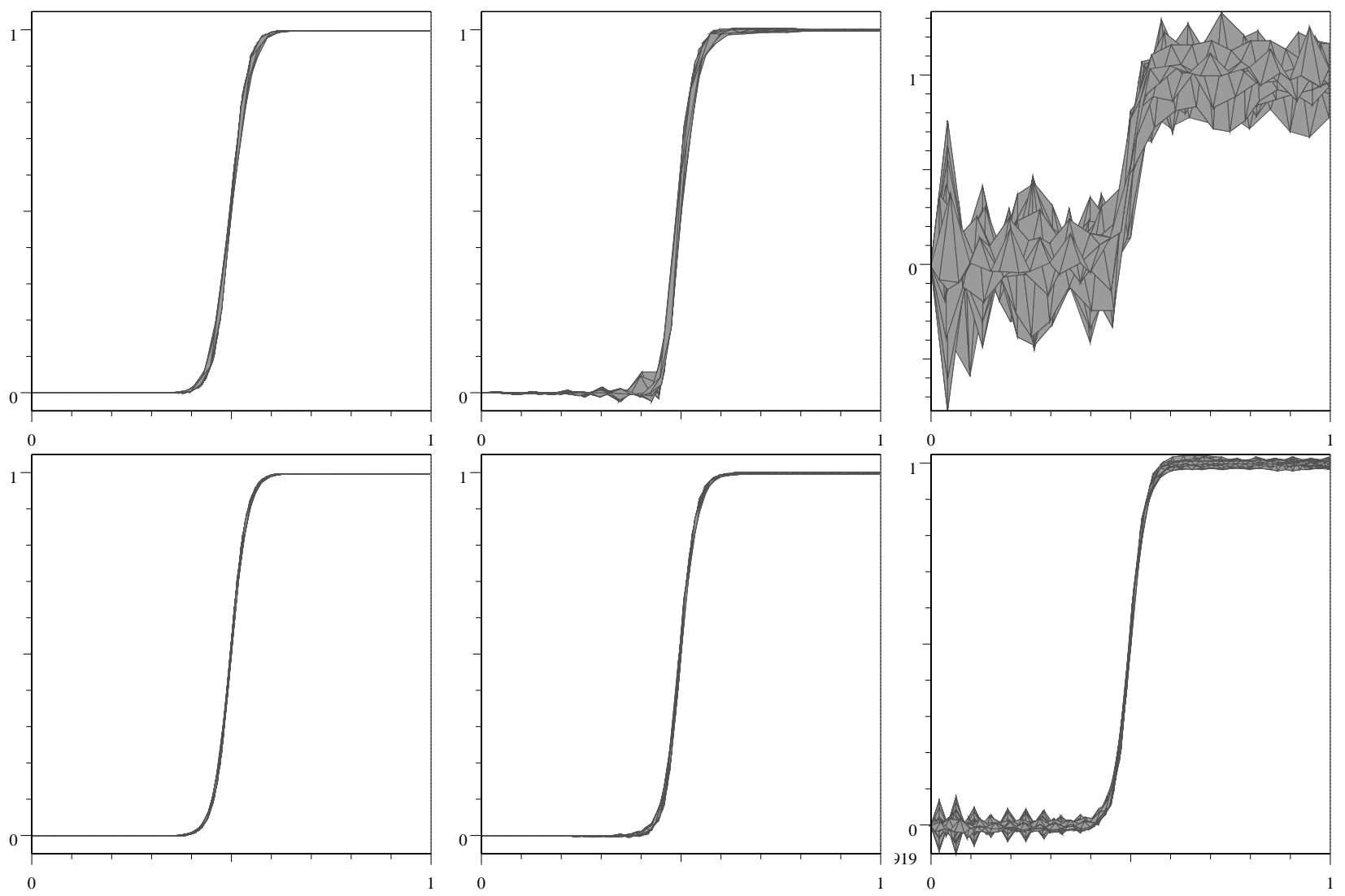

Figure 7. Problem: $\partial_{y} u=\frac{1}{2 \epsilon}\left(1-\left(\tanh \left(\frac{y-0.5}{\epsilon}\right)\right)^{2}\right)$. Top left: projection on plane $y=0$ of $\mathbb{P}_{1}$ interpolate of exact solution; top center: projection on plane $y=0$ of two-level $\mathbb{P}_{2}$ subgrid viscosity solution; top right: projection on plane $y=0$ of Galerkin solution; bottom left: projection on plane $y=0$ of $\mathbb{P}_{2}$ interpolate of exact solution; bottom center: projection on plane $y=0$ of two-level $\mathbb{P}_{2}$ subgrid viscosity solution; bottom right: projection on plane $y=0$ of $\mathbb{P}_{2}$ the Galerkin solution.

stabilized techniques by formally eliminating the small-scale functions. To clarify this point, let us denote by $A: X_{H} \longrightarrow \mathrm{L}^{2}(\Omega)$ so that $A u_{H \mid T_{H}}=\sigma u_{H}+\beta \cdot \nabla u_{H}$. Let $G_{h}^{H}: \mathrm{L}^{2}(\Omega) \longrightarrow X_{h}^{H}$ be so that for all $g \in \mathrm{L}^{2}(\Omega)$, $G_{h}^{H}(g)$ is the solution to the following problem

$$
\forall v_{h}^{H} \in X_{h}^{H}, \quad a\left(G_{h}^{H}(g), v_{h}^{H}\right)+b_{h}\left(G_{h}^{H}(g), v_{h}^{H}\right)=\int_{\Omega} g v_{h}^{H} .
$$

If $X_{h}^{H}$ is composed of bubble functions, this problem is very simple to solve since it amounts to solving on each simplex $T_{H}$ a linear system of dimension $k_{h}$, where recall that $k_{h}$ is the number of small-scale space basis functions on $\hat{T}$. However, for two-level approximations, the support of the subgrid functions interact; as a result, (6.1) cannot be be solved easily in practice. Though, theoretically we infer that $u_{H}$ is solution to the following problem:

$$
\forall v_{H} \in X_{H}, \quad a\left(u_{H}, v_{H}\right)+a\left(G_{h}^{H}\left(f-A u_{H}\right), v_{H}\right)=\int_{\Omega} f v_{H}
$$


Hence, the method that we propose theoretically amounts to stabilizing the Galerkin method by adding a term that is proportional to the residual of the equation, namely $f-A u_{H}$. That this condensed form of the problem is more practical in terms of programming effort than (3.9) for quite general problems is not clear. Though, from the author's experience, this is not true for the two-level approximations presented in this paper.

The proof of convergence given in the present paper assume that the grid is quasi-uniform since uniform inverse inequalities are used. This hypothesis can be weakened by using the local meshsize in the definition of the artificial diffusion bilinear form $b_{h}$. This question together with some generalizations of the present technique to the approximation of non coercive bilinear forms in general Hilbert spaces will be treated in a forthcoming paper.

Some ideas developed in this paper where initiated by a discussion with Claude Bardos at an ASCI meeting. The author is also grateful to L. Quartapelle for helpful discussions and remarks that improved the content of this paper. The present work has been partly supported by ASCI (UPR-CNRS 9029), Orsay.

\section{REFERENCES}

[1] A.A.O. Ammi and M. Marion, Nonlinear Galerkin methods and mixed finite elements: two-grid algorithms for the NavierStokes equations. Numer. Math. 68 (1994) 189-213.

[2] D.N. Arnold, F. Brezzi and M. Fortin, A stable finite element for the Stokes equations. Calcolo 21 (1984) $337-344$.

[3] P. Azerad and G. Pousin, Inégalité de Poincaré courbe pour le traitement variationnel de l'équation de transport. C.R. Acad. Sci. Paris Sér. I 322 (1996) 721-727.

[4] C. Baiocchi, F. Brezzi and L.P. Franca, Virtual bubbles and Galerkin-least-square type methods (Ga.L.S). Comput. Methods Appl. Mech. Engrg. 105 (1993) 125-141.

[5] C. Bardos, Problèmes aux limites pour les équations aux dérivées partielles du premier ordre à coefficients réels; théorèmes d'approximation; application à l'équation de transport. Ann. Sci. École Norm. Sup. Sér. IV 3 (1970) 185-233.

[6] M. Barton-Smith, Mémoire de DEA. Analyse Numérique, Paris XI, Internal report LIMSI (1999).

[7] F. Brezzi, M.O. Bristeau, L. Franca, M. Mallet and G. Rogé, A relationship between stabilized finite element methods and the Galerkin method with bubble functions. Comput. Methods Appl. Mech. Engrg. 96 (1992) 117-129.

[8] A.N. Brooks and T.J.R. Hughes, Streamline Upwind/Petrov-Galerkin formulations for convective dominated flows with particular emphasis on the incompressible Navier-Stokes equations. Comput. Methods Appl. Mech. Engrg. 32 (1982) 199-259.

[9] M. Crouzeix and P.-A. Raviart, Conforming and nonconforming finite element methods for solving the stationary Stokes equations. RAIRO Anal. Numér. 7 (1973) 33-76.

[10] R. Codina, Comparison of some finite element methods for solving the diffusion-convection-reaction equations. Comput. Methods Appl. Mech. Engrg. 156 (1997) 185-210.

[11] J. Douglas and T.F. Russell, Numerical methods for convection dominated diffusion problems based on combining the method of characteristics with finite element methods or finite difference method. SIAM J. Numer. Anal. 19 (1982) 871-885.

[12] R. Dautray and J.-L. Lions, Analyse mathématique et calcul numérique pour les sciences et les techniques. Masson, Paris (1984).

[13] C. Foias, O.P. Manley and R. Temam, Modelization of the interaction of small and large eddies in two dimensional turbulent flows. Math. Modelling Numer. Anal. 22 (1988) 93-114.

[14] M. Fortin, An analysis of the convergence of mixed Finite Element Methods. RAIRO Anal. Numér. 11 (1977) 341-354.

[15] L.P. Franca and C. Farhat, Bubble functions prompt unusual stabilized finite element methods. Comput. Methods Appl. Mech. Engrg. 123 (1994) 299-308.

[16] M. Germano, U. Piomelli, P. Moin and W.H. Cabot, A dynamic subgrid-scale eddy viscosity model. Phys. Fluids A 3 (1991) $1760-1765$.

[17] V. Girault and P.-A. Raviart, Finite Element Methods for Navier-Stokes Equations. Springer Ser. Comput. Math. 5, SpringerVerlag (1986).

[18] J.-L. Guermond, Stabilisation par viscosité de sous-maille pour l'approximation de Galerkin des opérateurs monotones. C.R. Acad. Sci. Paris Sér. I 328 (1999) 617-622.

[19] T.J.R. Hughes, Multiscale phenomena: Green's function, the Dirichlet-to-Neumann formulation, subgrid scale models, bubbles and the origins of stabilized formulations. Comput. Methods Appl. Mech. Engrg. 127 (1995) 387-401.

[20] C. Johnson, U. Nävert, J. Pitkäranta, Finite element methods for linear hyperbolic equations. Comput. Methods Appl. Mech. Engrg. 45 (1984) 285-312.

[21] O.A. Ladyzhenskaya, Modification of the Navier-Stokes equations for large velocity gradients. Boundary Value Problems of Mathematical Physics and Related Aspects of Function Theory, Consultants bureau, New-York (1970).

[22] M. Marion and R. Temam, Nonlinear Galerkin methods: the finite element case. Numer. Math. 57 (1990) 1-22. 
[23] T.E. Peterson, A note on the convergence of the discontinuous Galerkin method for a scalar hyperbolic equation. SIAM $J$. Numer. Anal. 28 (1991) 133-140.

[24] O. Pironneau, On the transport-diffusion algorithm and its applications to the Navier-Stokes equations. Numer. Math. 38 (1982) 309-332.

[25] A. Quarteroni and A. Valli, Numerical Approximation of Partial Differential Equations. Springer Ser. Comput. Math. 23, Springer-Verlag (1994).

[26] J. Smagorinsky, General circulation experiments with the primitive equations. I. The basic experiments. J. Atmospheric Sci. 32 (1963) 680-689.

[27] E. Süli, Convergence and non-linear stability of the Lagrange-Galerkin method for the Navier-Stokes equations. Numer. Math. 53 (1988) 459-483.

[28] B. García-Archilla, J. Novo and E.S. Titi, Postprocessing the Galerkin method: a novel approach to Approximate Inertial Manifolds. SIAM J. Numer. Anal. 35 (1998) 941-972.

[29] G. Zhou, How accurate is the streamline diffusion finite element method? Math. Comp. 66 (1997) 31-44. 